\title{
Low-mass protostars and dense cores in different evolutionary stages in IRAS 00213+6530
}

\author{
G. Busquet ${ }^{1}$, Aina Palau ${ }^{1,2}$, R. Estalella ${ }^{1}$, J. M. Girart ${ }^{3}$, G. Anglada ${ }^{4}$, and I. Sepúlveda ${ }^{1}$ \\ 1 Departament d'Astronomia i Meteorologia (IEEC-UB), Institut de Ciències del Cosmos, Universitat de Barcelona, \\ Martí i Franquès 1, 08028 Barcelona, Catalunya, Spain \\ e-mail: gbusquet@am.ub.es \\ 2 Centro de Astrobiología (CSIC-INTA), Laboratorio de Astrofísica Estelar y Exoplanetas, LAEFF campus, PO Box 78, \\ 28691 Villanueva de la Cañada (Madrid), Spain \\ 3 Institut de Ciències de l'Espai (CSIC-IEEC), Campus UAB, Facultat de Ciències, Torre C-5 parell, 08193 Bellaterra, Catalunya, \\ Spain \\ 4 Instituto de Astrofísica de Andalucía (CSIC), C/ Camino Bajo de Huétor 50, 18008, Granada, Spain
}

Received 13 February 2009 / Accepted 27 July 2009

\section{ABSTRACT}

\begin{abstract}
Aims. The aim of this paper is to study with high angular resolution a dense core associated with a low-luminosity IRAS source, IRAS 00213+6530, in order to investigate whether low mass star formation is taking place in isolation.

Methods. We carried out observations at $1.2 \mathrm{~mm}$ with the IRAM $30 \mathrm{~m}$ telescope, and VLA observations in the continuum mode at $6 \mathrm{~cm}, 3.6 \mathrm{~cm}, 1.3 \mathrm{~cm}$ and $7 \mathrm{~mm}$, together with $\mathrm{H}_{2} \mathrm{O}$ maser and $\mathrm{NH}_{3}$ lines toward IRAS $00213+6530$. Additionally, we observed the CCS $J_{N}=2{ }_{1}-1_{0}$ transition, and $\mathrm{H}_{2} \mathrm{O}$ maser emission using the NASA $70 \mathrm{~m}$ antenna at Robledo de Chavela, Spain. We studied the nature of the centimeter and millimeter emission of the young stellar objects (YSOs) found in the region, and the physical properties of the dense gas and dust emission.

Results. The centimeter and millimeter continuum emission, together with the near infrared data from 2MASS allowed us to identify three YSOs, IRS 1, VLA 8A, and VLA 8B, with different radio and infrared properties, and which seem to be in different evolutionary stages. IRS 1 , detected only in the infrared, is in the more advanced stage. On the other hand, VLA 8A, bright at centimeter and millimeter wavelengths, coincides with a near infrared 2MASS source, whereas VLA 8B has no infrared emission associated with it and is in the earliest evolutionary stage. The overall structure of the $\mathrm{NH}_{3}$ emission consists of three clouds. Two of these, MM1 and MM2, are associated with dust emission at millimeter wavelengths, while the southern cloud is only detected in $\mathrm{NH}_{3}$. The YSOs are embedded in MM1, where we found evidence of line broadening and temperature enhancements. On the other hand, the southern cloud and MM2 appear to be quiescent and starless. Concerning the $1.2 \mathrm{~mm}$ dust emission, we modeled the radial intensity profile of MM1. The model fits the data reasonably well, but it underestimates the intensity at small projected distances from the $1.2 \mathrm{~mm}$ peak, probably due to the presence of multiple YSOs embedded in the dusty envelope. There is a strong differentiation in the relative $\mathrm{NH}_{3}$ abundance with low values of $\sim 2 \times 10^{-8}$ toward MM1, which harbors the YSOs, and high values, up to $10^{-6}$, toward the southern cloud and MM2, suggesting that these clouds could be in a young evolutionary stage.

Conclusions. IRAS $00213+6530$ harbors a multiple system of low-mass protostars, indicating that star formation in this cloud is taking place in groups or clusters, rather than in isolation. The low-mass YSOs found in IRAS $00213+6530$ are in different evolutionary stages, suggesting that star formation takes place in different episodes.
\end{abstract}

Key words. stars: formation - ISM: individual objects: IRAS 00213+6530 - ISM: clouds

\section{Introduction}

It is widely accepted that there are two modes of star formation: the isolated mode and the clustered mode. This classification results from studying the association between dense cores and young stellar objects (YSOs). For example, Benson \& Myers (1989) study a wide sample of ammonia cores and its relation with the position of IRAS sources, and find that typically only one IRAS source is associated with a single ammonia core in the Taurus Molecular Cloud, where star formation can be assumed to take place in isolation. On the contrary, Orion and Perseus would be examples of molecular clouds forming stars in clustered mode (Lada et al. 1993). A broad base of recent studies, carried out with higher angular resolution than that of Benson \& Myers, show that most stars form in groups or clusters (e.g., Clarke et al. 2000; Lada \& Lada 2003), including low-mass stars (e.g., Gómez et al. 1993; Huard et al. 1999; Lee et al. 2006;
Brooke et al. 2007; Teixeira et al. 2007), indicating that truly isolated star formation is rare. Adams \& Myers (2001) propose an intermediate case between the isolated and clustered modes, i.e., star formation in groups, and propose that most stars form in groups and/or clusters, i.e., in cluster environments. However, the theories of low mass star formation assume that star formation takes place in the isolated mode (Shu et al. 1987; Lada 1999). Since star formation in cluster environments may differ from the isolated mode (e.g., Pfalzner et al. 2008), it is necessary to study with high angular resolution dense cores associated with one single IRAS source to assess if star formation is really taking place in isolation. In this context, we aim at investigating with high angular resolution a dense ammonia core associated with a single low luminosity IRAS source, IRAS 00213+6530 (hereafter I00213).

I00213, with a luminosity of $\lesssim 20 L_{\odot}$ and at 850 pc of distance, belongs to the molecular cloud M120.1+3.0 
Table 1. VLA observational parameters in the IRAS $00213+6530$ region.

\begin{tabular}{|c|c|c|c|c|c|c|c|c|c|c|}
\hline \multirow[b]{2}{*}{ Transition } & \multirow[b]{2}{*}{$\begin{array}{c}\lambda \\
(\mathrm{cm})\end{array}$} & \multicolumn{2}{|c|}{ Phase Center } & \multirow[b]{2}{*}{$\begin{array}{c}\text { Phase } \\
\text { Calibrator }\end{array}$} & \multirow{2}{*}{$\begin{array}{l}\text { Bootstrapped } \\
\text { Flux Density } \\
(\mathrm{Jy})\end{array}$} & \multicolumn{2}{|c|}{ Synthesized Beam } & \multicolumn{3}{|c|}{ On-Source } \\
\hline & & $\begin{array}{c}\alpha(\mathrm{J} 2000) \\
(\mathrm{h} \mathrm{m} \mathrm{s})\end{array}$ & $\begin{array}{c}\delta(\mathrm{J} 2000) \\
\left({ }^{\circ},{ }^{\prime} \prime\right)\end{array}$ & & & $\begin{array}{l}\text { HPBW } \\
(\operatorname{arcsec})\end{array}$ & $\begin{array}{c}\text { PA } \\
(\mathrm{deg})\end{array}$ & $\begin{array}{c}\text { rms Noise } \\
\left(\mathrm{mJy} \mathrm{beam}^{-1}\right)\end{array}$ & $\begin{array}{c}\text { Time } \\
\text { (hours) }\end{array}$ & Epoch \\
\hline continuum & 6 & 002410.41 & +654702.0 & $0014+612$ & $1.940 \pm 0.006$ & $17.9 \times 11.7$ & 54 & 0.024 & 1.2 & 2000 \\
\hline continuum & 3.6 & 002410.41 & +654702.0 & $0014+612$ & $1.281 \pm 0.010$ & $21.2 \times 9.2$ & -68 & 0.023 & 1.0 & 2000 \\
\hline continuum & 3.6 & 002411.44 & +654709.6 & $0019+734$ & $1.070 \pm 0.003$ & $9.9 \times 7.2$ & 55 & 0.027 & 0.5 & 2004 \\
\hline continuum & 1.3 & 002411.44 & +65 4709.6 & $0019+734$ & $2.41 \pm 0.08$ & $1.5 \times 1.0$ & 32 & 0.15 & 0.3 & 2006 \\
\hline continuum & 0.7 & 002411.44 & +654709.6 & $0019+734$ & $1.71 \pm 0.06$ & $2.9 \times 2.6$ & -89 & 0.19 & 1.0 & 2004 \\
\hline $\mathrm{NH}_{3}(1,1)$ & 1.3 & 002411.44 & +654709.6 & $0019+734$ & $1.58 \pm 0.05$ & $4.1 \times 3.7$ & -52 & $1.3^{a}$ & 6 & 2004 \\
\hline $\mathrm{NH}_{3}(2,2)$ & 1.3 & 002411.44 & +65 4709.6 & $0019+734$ & $1.58 \pm 0.05$ & $4.1 \times 3.7$ & -51 & $1.1^{a}$ & 6 & 2004 \\
\hline $\mathrm{H}_{2} \mathrm{O} 6_{16}-5_{23}$ & 1.3 & 002411.44 & +65 4709.6 & $0019+734$ & $2.41 \pm 0.08$ & $2.3 \times 1.4$ & 17 & $3.0^{a}$ & 0.3 & 2006 \\
\hline
\end{tabular}

${ }^{a}$ Per channel.

(Yang et al. 1990) in the Cepheus OB4 star-forming region. The region is physically related to the $\mathrm{H}$ II region S171 (Yang et al. 1990). The $\mathrm{NH}_{3}$ emission in the north of M120.1+3.0 was studied through single-dish observations by Sepúlveda (2001). The $\mathrm{NH}_{3}$ emission consists of two condensations, each one peaking very close to the position of an IRAS source, I00213 and IRAS 00217+6533 (I00217), suggesting that both IRAS sources are deeply embedded in high density gas. The mass derived for the condensation associated with I00213 is $\geq 45 M_{\odot}$. The ammonia emission engulfing both IRAS sources is associated with $\mathrm{CO}$ high-velocity emission, indicating the presence of a molecular outflow in the region (Yang et al. 1990). However, it is not clear which IRAS source is driving the outflow.

In this paper we report on high angular resolution observations with the Very Large Array (VLA) of the continuum emission at $6 \mathrm{~cm}, 3.6 \mathrm{~cm}, 1.3 \mathrm{~cm}$, and $7 \mathrm{~mm}$, as well as of the dense gas traced by $\mathrm{NH}_{3}(1,1)$ and $\mathrm{NH}_{3}(2,2)$ together with observations of $\mathrm{H}_{2} \mathrm{O}$ maser emission. In addition we also present the continuum emission at $1.2 \mathrm{~mm}$ observed with the IRAM $30 \mathrm{~m}$ telescope, and $\mathrm{CCS}$ and $\mathrm{H}_{2} \mathrm{O}$ maser observations carried out with the NASA $70 \mathrm{~m}$ antenna at Robledo de Chavela. The paper layout is the following: in Sect. 2 we describe our observations and the data reduction process, and present the main results for the continuum and molecular line emission in Sect. 3. In Sect. 4 we analyze the dust and $\mathrm{NH}_{3}$ emission and show the method used to derive the $\mathrm{NH}_{3}$ abundance in this region. Finally, in Sect. 5 we discuss our findings, and we list the main conclusions in Sect. 6.

\section{Observations}

\subsection{IRAM 30 m observations}

The MPIfR 37-element bolometer array MAMBO at the IRAM $30 \mathrm{~m}^{\text {telescope }}{ }^{1}$ was used to map the $1.2 \mathrm{~mm}$ dust continuum emission toward I00213. The observations were carried out on 2006 June 2. The main beam has a HPBW of $10^{\prime \prime}$. We used the on-the-fly mapping mode, in which the telescope scans continuously in azimuth along each row. The sampled area was $200^{\prime \prime} \times 140^{\prime \prime}$, and the scanning speed was $5^{\prime \prime} \mathrm{s}^{-1}$. Each scan was separated by $5^{\prime \prime}$ in elevation. The secondary mirror was wobbling at a rate of $2 \mathrm{~Hz}$ in azimuth with a wobbler throw of $46^{\prime \prime}$. The average zenith opacity was in the range $0.3-0.4$. Pointing and focus were done on NGC 7538. The rms of the final map was $\sim 3.8 \mathrm{mJy}$ beam $^{-1}$. Data reduction was performed with the

${ }^{1}$ IRAM is supported by INSU/CNRS (France), MPG (Germany), and IGN (Spain).
MOPSIC ${ }^{2}$ software package that contains the necessary scripts for data reduction (distributed by R. Zylcka).

\subsection{VLA radio continuum observations}

The observations were carried out using the VLA of the NRAO ${ }^{3}$ in the D configuration in the continuum mode at $6 \mathrm{~cm}$ and $3.6 \mathrm{~cm}$ on 2000 September 23, and at $3.6 \mathrm{~cm}$ and $7 \mathrm{~mm}$ during 2004 August 24. The observational parameters for each epoch are summarized in Table 1. During the first epoch, absolute flux calibration was achieved by observing $3 \mathrm{C} 286$, with an adopted flux density of $7.49 \mathrm{Jy}$ at $6 \mathrm{~cm}$ and $5.18 \mathrm{Jy}$ at $3.6 \mathrm{~cm}$. The absolute flux calibrator was $00137+331$ (3C 48) during the 2004 observations, for which flux densities of $3.15 \mathrm{Jy}$ and $0.53 \mathrm{Jy}$ were adopted at $3.6 \mathrm{~cm}$ and $7 \mathrm{~mm}$, respectively. In order to minimize the effects of atmospheric phase fluctuations, at $7 \mathrm{~mm}$ we used the fast switching technique (Carilli \& Holdaway 1997) between the source and the phase calibrator over a cycle of $80 \mathrm{~s}$, with $50 \mathrm{~s}$ spent on the source and $30 \mathrm{~s}$ on the calibrator. The $1.3 \mathrm{~cm}$ continuum emission was observed on 2006 December 2 simultaneously with the $\mathrm{H}_{2} \mathrm{O}$ maser emission (see below, Sect. 2.3).

Calibration and data reduction were performed using standard procedures of the Astronomical Imaging Processing System (AIPS) ${ }^{4}$ of the NRAO. Clean maps at $3.6 \mathrm{~cm}$ and $7 \mathrm{~mm}$ were made using the task IMAGR of AIPS with the robust parameter of Briggs (1995) set equal to 5, which is close to natural weighting, whereas the map at $6 \mathrm{~cm}$ was made with the robust parameter equal to zero in order to obtain a synthesized beam similar to that at $3.6 \mathrm{~cm}$. Since the signal-to-noise ratio of the longest baselines of the $7 \mathrm{~mm}$ data was low, we applied a $u v$ taper function of $80 \mathrm{k} \lambda$ in order to improve the sensitivity.

\section{3. $\mathrm{VLA} \mathrm{NH} \mathrm{H}_{3}$ and $\mathrm{H}_{2} \mathrm{O}$ maser observations}

The observations of $(J, K)=(1,1)$ and $(J, K)=(2,2)$ inversion lines of the ammonia molecule were carried out in the same run as the 2004 continuum observations. In Table 1 we summarize the observational parameters. The adopted flux density of the absolute flux calibrator $0137+331$ (3C 48) was $1.05 \mathrm{Jy}$ at a wavelength of $1.3 \mathrm{~cm}$, and the bandpass calibrator used

\footnotetext{
${ }^{2}$ See

http://www.iram.es/IRAMES/mainWiki/CookbookMopsic

3 The Very Large Array (VLA) is operated by the National Radio Astronomy Observatory (NRAO), a facility of the National Science Foundation operated under cooperative agreement by Associated Universities, Inc.

${ }^{4}$ See http://aips.nrao.edu
} 
was $0319+415$ (3C 84). We used the 4IF spectral line mode, which allows simultaneous observations of the $\mathrm{NH}_{3}(1,1)$ and $(2,2)$ lines with two polarizations for each line. The bandwidth used was $3.12 \mathrm{MHz}$, with 63 channels with a channel spacing of $48.8 \mathrm{kHz}\left(0.6 \mathrm{~km} \mathrm{~s}^{-1}\right.$ at $\left.1.3 \mathrm{~cm}\right)$ centered at $v_{\mathrm{LSR}}=-19.0 \mathrm{~km} \mathrm{~s}^{-1}$, plus a continuum channel that contains the average of the central $75 \%$ of the bandwidth.

The water maser line at $22.2351 \mathrm{GHz}\left(6_{16}-5_{23}\right.$ transition) was observed with the VLA in the $\mathrm{C}$ configuration during 2006 December 2. The phase center was the same as for the $\mathrm{NH}_{3}$ observations, and the adopted flux density of the absolute flux calibrator, $0137+331$ (3C 48), was $1.13 \mathrm{Jy}$ at $1.3 \mathrm{~cm}$. We summarized the observational parameters in Table 1 . We used the 4IF mode, employing two IF with a total bandwidth of $3.12 \mathrm{MHz}$, with 63 channels with a channel spacing of $48.8 \mathrm{kHz}$ $\left(0.6 \mathrm{~km} \mathrm{~s}^{-1}\right)$ centered at $-10 \mathrm{~km} \mathrm{~s}^{-1} 5$, and two IF with a total bandwidth of $25 \mathrm{MHz}$ were used to observe the continuum emission.

The $\mathrm{NH}_{3}$ and $\mathrm{H}_{2} \mathrm{O}$ data were reduced with the standard AIPS procedures. The images were constructed using natural weighting in both cases.

\subsection{NASA $70 \mathrm{~m} \mathrm{CCS} \mathrm{and} \mathrm{H}_{2} \mathrm{O}$ maser observations}

We carried out a $\mathrm{H}_{2} \mathrm{O}$ maser emission monitoring toward IRAS 00213+6530 with the NASA $70 \mathrm{~m}$ antenna (DSS-63) at Robledo de Chavela (Spain). The observations were performed in 2008 Apr 18, June 19, and September 23 using a cooled highelectron-mobility transistor as $1.3 \mathrm{~cm}$ front-end, and a 384 channel spectrometer as backend, covering a bandwidth of $16 \mathrm{MHz}$ ( $216 \mathrm{~km} \mathrm{~s}^{-1}$ with $0.6 \mathrm{~km} \mathrm{~s}^{-1}$ resolution). Spectra were taken in position-switching mode. The HPBW of the telescope at this frequency is $\sim 41^{\prime \prime}$. The typical system temperature was $120 \mathrm{~K}$ and the total integration time was around $30 \mathrm{~min}$ (on+off) for each session.

In addition to the $\mathrm{H}_{2} \mathrm{O}$ maser observations, we also observed the CCS $J_{N}=21_{1}-1_{0}$ transition $(22.344 \mathrm{GHz})$, with the same bandwidth and spectral resolution used for $\mathrm{H}_{2} \mathrm{O}$. The CCS transition was observed on October 3rd 2008 and on February 7th 2009 during a total integration time of 26 and 40 minutes, respectively. The system temperature was $60 \mathrm{~K}$ and $73 \mathrm{~K}$, respectively.

For all the observations, the rms pointing accuracy of the telescope was better than $10^{\prime \prime}$. A noise diode was used to calibrate the data, and the uncertainty in the flux calibration is estimated to be $\sim 30 \%$. The data reduction was performed using the CLASS package, which is part of the GILDAS ${ }^{6}$ software.

\section{Results}

\subsection{Continuum at $1.2 \mathrm{~mm}$}

Figure 1 shows the $1.2 \mathrm{~mm}$ continuum emission observed with the IRAM $30 \mathrm{~m}$ telescope toward I00213. The overall structure of the dust emission consists of a central and compact dust condensation, MM1, with some extended structure to the west. A 2D Gaussian fit to MM1 yields a deconvolved size of $13^{\prime \prime} \times 12^{\prime \prime}$, $\mathrm{PA}=92^{\circ}$. In addition, we also detected a fainter dust condensation, MM2, located to the northwest of MM1, and elongated in

\footnotetext{
${ }^{5}$ Note that the center velocity for the $\mathrm{H}_{2} \mathrm{O}$ maser line observed with the VLA is shifted by $9 \mathrm{~km} \mathrm{~s}^{-1}$ from that of the $\mathrm{NH}_{3}$ observations since the $\mathrm{H}_{2} \mathrm{O}$ maser reported by Han et al. (1998) was detected at $v_{\mathrm{LSR}}=$ $-0.7 \mathrm{~km} \mathrm{~s}^{-1}$.

${ }^{6}$ See http://www. iram. fr/IRAMFR/GILDAS
}

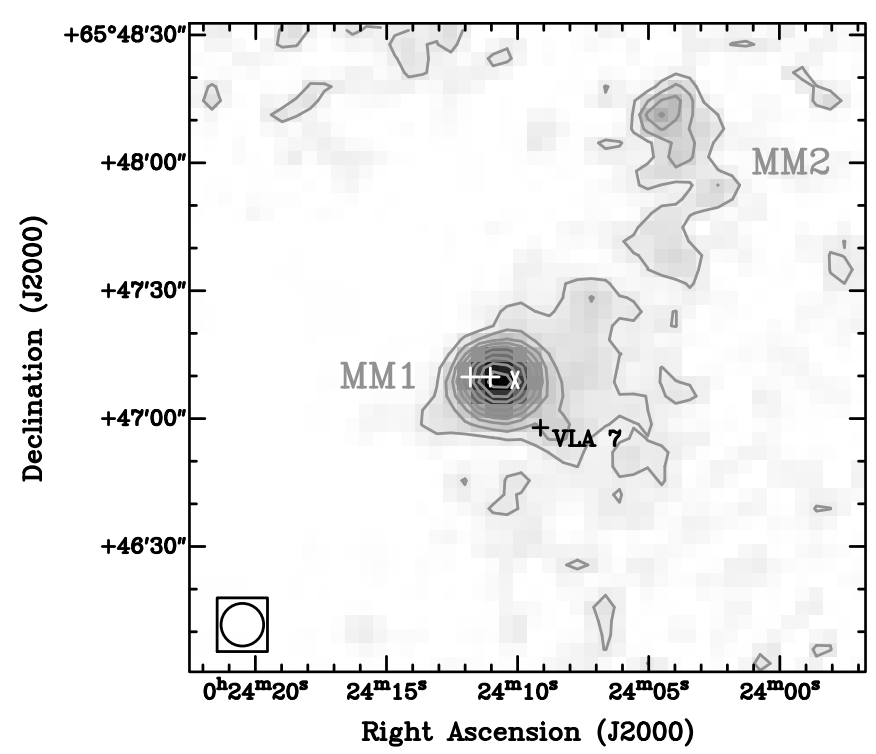

Fig. 1. $1.2 \mathrm{~mm}$ continuum emission toward IRAS 00213+6530. Contour levels are $3,6,9,12,15,20,25,30$ and 35 times the rms of the map, $3.8 \mathrm{mJy}^{\text {beam }}{ }^{-1}$. The synthesized beam of the IRAM $30 \mathrm{~m}$ is shown in the bottom left corner of the image. White crosses indicate the position of the millimeter sources VLA 8A and VLA 8B, and the white tilted cross marks the position of IRS 1. VLA 7 is indicated by the black cross (see Sect. 3.2 for the objects labeled in this figure).

Table 2. Parameters of the $1.2 \mathrm{~mm}$ emission.

\begin{tabular}{ccccc}
\hline \hline & \multicolumn{2}{c}{ Position } & & \\
\cline { 2 - 3 } & $\alpha(\mathrm{J} 2000)$ & $\delta(\mathrm{J} 2000)$ & & $I_{v}^{\text {peak }}$ \\
$(\mathrm{h} \mathrm{m} \mathrm{s})$ & $\left({ }^{\circ}{ }^{\prime \prime}{ }^{\prime \prime}\right)$ & $\left(\mathrm{mJy} \mathrm{beam}^{-1}\right)$ & $\begin{array}{c}S_{v} \\
(\mathrm{mJy})\end{array}$ \\
\hline MM1 & 002410.7 & 654709 & 146 & $332 \pm 35$ \\
MM2 & 002404.4 & 654812 & 46 & $146 \pm 9$ \\
\hline
\end{tabular}

the north-south direction, in a filamentary structure connecting MM1 and MM2. The main results are summarized in Table 2.

\subsection{VLA radio continuum emission}

We detected 11 sources at $6 \mathrm{~cm}$, and 7 sources at $3.6 \mathrm{~cm}$ above the $4 \sigma$ detection threshold. Figure 2 shows the $6 \mathrm{~cm}$ and $3.6 \mathrm{~cm}$ continuum emission observed with the VLA toward I00213. In Table 3 we list the positions and flux densities, corrected for primary beam response, of the detected sources, and the estimated spectral indices between $6 \mathrm{~cm}$ and $3.6 \mathrm{~cm}$. To obtain the $3.6 \mathrm{~cm}$ map we analyzed separately the observations of the two epochs (see Sect. 2.2) in order to see the degree of variability of the detected sources at this wavelength. The final map was obtained after subtracting the $u v$ data of VLA 3, which presents a variability greater than $8 \sigma$, and VLA 6, detected in 2004 but not during the observations carried out in 2000. In Table 4 we show the flux density measured in 2000 and 2004 for VLA 3 and VLA 6, as well as the variability during this period. Both sources, VLA 3 and VLA 6, have a negative spectral index (from simultaneous observations at $6 \mathrm{~cm}$ and $3.6 \mathrm{~cm}$ ) and are probably non-thermal extragalactic background sources.

As can be seen in Fig. 3 (middle panel), at $3.6 \mathrm{~cm}$ we detected two sources toward I00213, VLA 7 and VLA 8, separated by $20^{\prime \prime}$ and both inside the position error ellipse of the IRAS source. These sources are barely resolved at $6 \mathrm{~cm}$, with VLA 8 just being a weak prolongation to the north-east of VLA 7 (see Fig. 3 top panel). Yet, the higher angular resolution of the $3.6 \mathrm{~cm}$ 
Table 3. Parameters of the continuum sources detected in the IRAS $00213+6530$ region.

\begin{tabular}{|c|c|c|c|c|c|}
\hline \multirow[b]{2}{*}{ Source } & \multicolumn{2}{|c|}{ Position ${ }^{\mathrm{a}}$} & \multirow{2}{*}{$\begin{array}{c}\text { Flux Density } \\
\text { at } 6 \mathrm{~cm} \\
(\mathrm{mJy})\end{array}$} & \multirow{2}{*}{$\begin{array}{c}\text { Flux Density } \\
\text { at } 3.6 \mathrm{~cm} \\
(\mathrm{mJy})\end{array}$} & \multirow{2}{*}{$\begin{array}{c}\text { Spectral } \\
\text { Index } \\
6 \mathrm{~cm}-3.6 \mathrm{~cm}\end{array}$} \\
\hline & $\begin{array}{c}\alpha(\mathrm{J} 2000) \\
(\mathrm{h} \mathrm{m} \mathrm{s})\end{array}$ & $\begin{array}{c}\delta(\mathrm{J} 2000) \\
\left({ }^{\circ}{ }^{\prime}{ }^{\prime \prime}\right)\end{array}$ & & & \\
\hline 1 & 002321.01 & +654346.5 & $0.87 \pm 0.07$ & $\ldots{ }^{b}$ & \\
\hline 2 & 002345.65 & +654836.1 & $0.17 \pm 0.03$ & $<0.18^{c}$ & $<0.10$ \\
\hline 3 & 002346.41 & +654912.7 & $1.66 \pm 0.04$ & $0.38 \pm 0.07^{d}$ & $-2.7 \pm 0.3$ \\
\hline 4 & 002349.01 & +654614.7 & $0.69 \pm 0.03$ & $0.42 \pm 0.02$ & $-0.9 \pm 0.1$ \\
\hline 5 & 002352.06 & +653958.9 & $4.20 \pm 0.60$ & $\ldots{ }^{b}$ & \\
\hline 6 & 002353.70 & +654941.6 & $0.86 \pm 0.03$ & $<0.25^{c, d}$ & $<-2.1$ \\
\hline 7 & 002409.11 & +654658.0 & $0.57 \pm 0.03$ & $0.24 \pm 0.02$ & $-1.6 \pm 0.2$ \\
\hline 8 & 002411.39 & +65 4709.6 & $0.16 \pm 0.03$ & $0.21 \pm 0.02$ & $0.5 \pm 0.4$ \\
\hline 9 & 002424.46 & +65 4905.9 & $0.13 \pm 0.03$ & $0.27 \pm 0.02$ & $1.3 \pm 0.4$ \\
\hline 10 & 002436.71 & +65 4827.5 & $0.55 \pm 0.03$ & $0.22 \pm 0.03$ & $-1.7 \pm 0.3$ \\
\hline 11 & 002456.70 & +654910.3 & $0.63 \pm 0.05$ & $\ldots{ }^{b}$ & $\ldots$ \\
\hline 12 & 002524.75 & +654548.3 & $4.90 \pm 1.10$ & $\ldots^{b}$ & \\
\hline
\end{tabular}

${ }^{a}$ Positions taken from the $3.6 \mathrm{~cm}$ map, except for non-detected sources at this band, for which positions correspond to the $6 \mathrm{~cm}$ map. ${ }^{b}$ Source well outside the primary beam at this wavelength. Primary beam correction greater than 12 , and the uncertainty in the corrected flux is very large. ${ }^{c}$ The upper limit for non-detected sources is $4 \sigma .{ }^{d}$ Flux density measured in 2000 September 23. Source highly variable, so to compute the spectral index we used observations carried out at the same epoch.
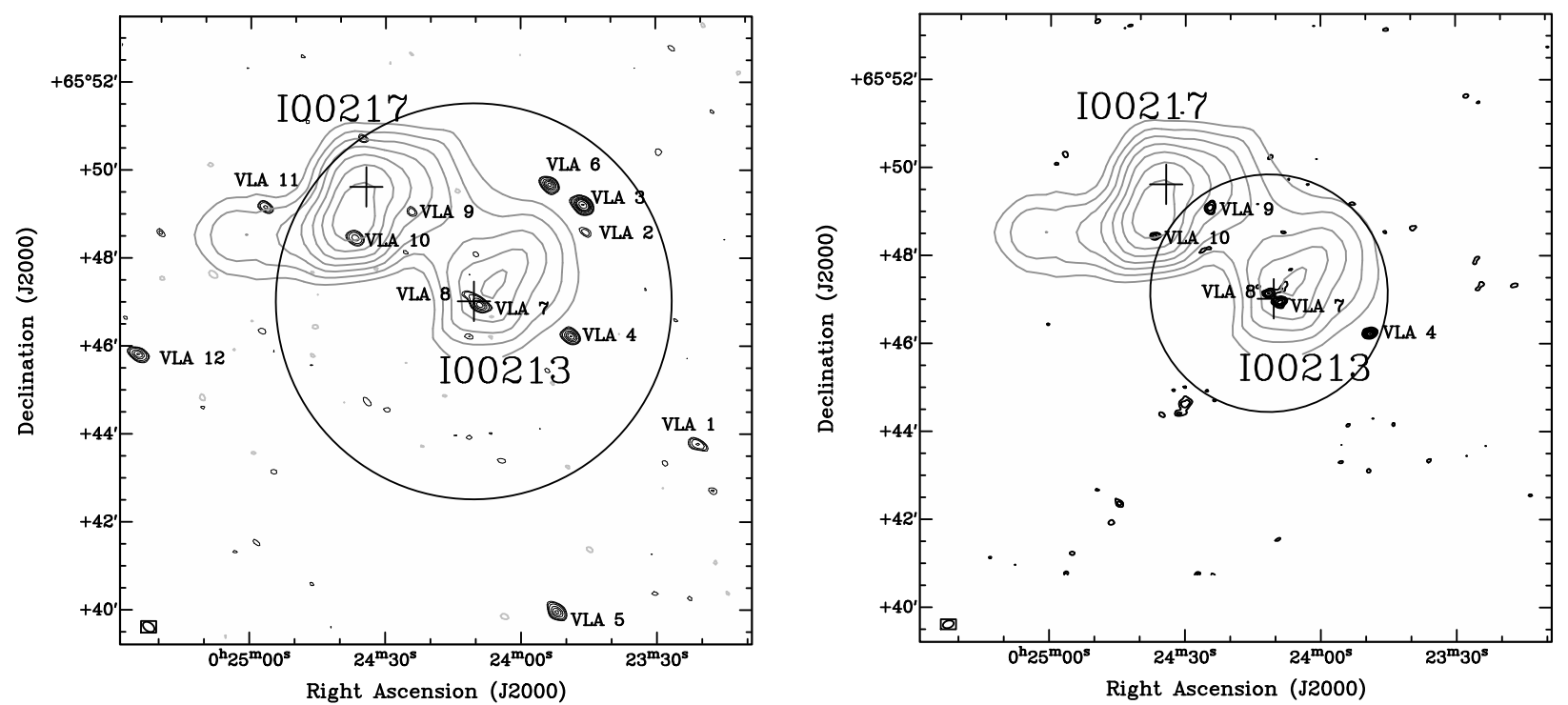

Fig. 2. In both panels grey contours represent the main beam brightness temperature of the main line of the $\mathrm{NH}_{3}(J, K)=(1,1)$ inversion transition from Sepúlveda (2001). Left: VLA $6 \mathrm{~cm}$ continuum emission map (black contours) of the I00213 region. Contour levels are $-3,3,4,6,10,14$, $18,22,32$, and 42 times the rms of the map, $24 \mu \mathrm{Jy}_{\text {beam }}^{-1}$. The synthesized beam, $17^{\prime \prime} .9 \times 11^{\prime \prime} 7$, with PA $=54^{\circ}$, is shown in the bottom left corner of the image. Right: VLA $3.6 \mathrm{~cm}$ continuum emission map (black contours) of the I00213 region. Contour levels are $-3,3,4,6,8,10$, and 12 times the rms of the map, $18 \mu \mathrm{Jy}_{\text {beam }}^{-1}$. The synthesized beam, $15^{\prime \prime} 14 \times 99^{\prime} .89$, with PA $=-72^{\circ}$, is shown in the bottom left corner of the image. The positions of the IRAS sources are indicated by crosses. IRAS $00213+6530$ is at the center of the figure, while IRAS $00217+6533$ is at the north-east. The VLA primary beam, $9^{\prime}$ at $6 \mathrm{~cm}$ and $5^{\prime}$ at $3.6 \mathrm{~cm}$ are also indicated by black circumferences.

Table 4. Highly variable sources at $3.6 \mathrm{~cm}$.

\begin{tabular}{|c|c|c|c|}
\hline Source & $\begin{array}{c}2000 \text { Obs. } \\
S_{v}(3.6 \mathrm{~cm}) \\
(\mathrm{mJy})\end{array}$ & $\begin{array}{c}2004 \text { Obs. } \\
S_{v}(3.6 \mathrm{~cm}) \\
(\mathrm{mJy})\end{array}$ & $\begin{array}{l}\text { Variability } \\
\text { with respect } \\
\text { to } 2004\end{array}$ \\
\hline VLA 3 & $0.38 \pm 0.07$ & $1.86 \pm 0.07$ & $79 \%(\sim 21 \sigma)$ \\
\hline VLA 6 & $<0.25^{a}$ & $0.41 \pm 0.05$ & $>39 \%(>3 \sigma)$ \\
\hline
\end{tabular}

${ }^{a}$ The upper limit for non-detected sources is $4 \sigma$.

map allows to separate the two sources. VLA 8 peaks close to the position of the dust condensation MM1, whereas VLA 7 lies $\sim 20^{\prime \prime}$ to the south-west, in the extended structure of the dust emission (see Fig. 1). Both sources are spatially resolved and VLA 8 shows a weak tail extending to the west. A 2D Gaussian fit to the two sources (excluding the weak tail of VLA 8) yields deconvolved sizes of 9 . $7 \times 2$.' $2\left(\mathrm{PA}=46^{\circ}\right)$, and $13^{\prime \prime} .4 \times 33^{\prime \prime} .1$ $\left(\mathrm{PA}=63^{\circ}\right)$ for VLA 7 and VLA 8, respectively. The spectral index in the $6-3.6 \mathrm{~cm}$ range of VLA 7 is $-1.6 \pm 0.2$, characteristic of non-thermal emission, whereas VLA 8 has a spectral index of $0.5 \pm 0.4$, which is consistent with free-free thermal emission from ionized gas that may be arising from a thermal radio jet. Positive spectral indices, i.e., $\alpha \gtrsim-0.1$, have been found to be associated with sources driving molecular outflows (e.g., Anglada et al. 1998; Beltrán et al. 2001). The 2MASS $K_{\mathrm{s}}$-band image shows two sources, 2MASS J00241110+6547095 and 2MASS J00241010+6547091, the first nearly coinciding with VLA 8 and the second, named IRS 1 , lying $\sim 6^{\prime \prime}$ to the west.

The maps of the $1.3 \mathrm{~cm}$ and $7 \mathrm{~mm}$ continuum emission obtained with natural weighting are shown in Fig. 3 (bottom 


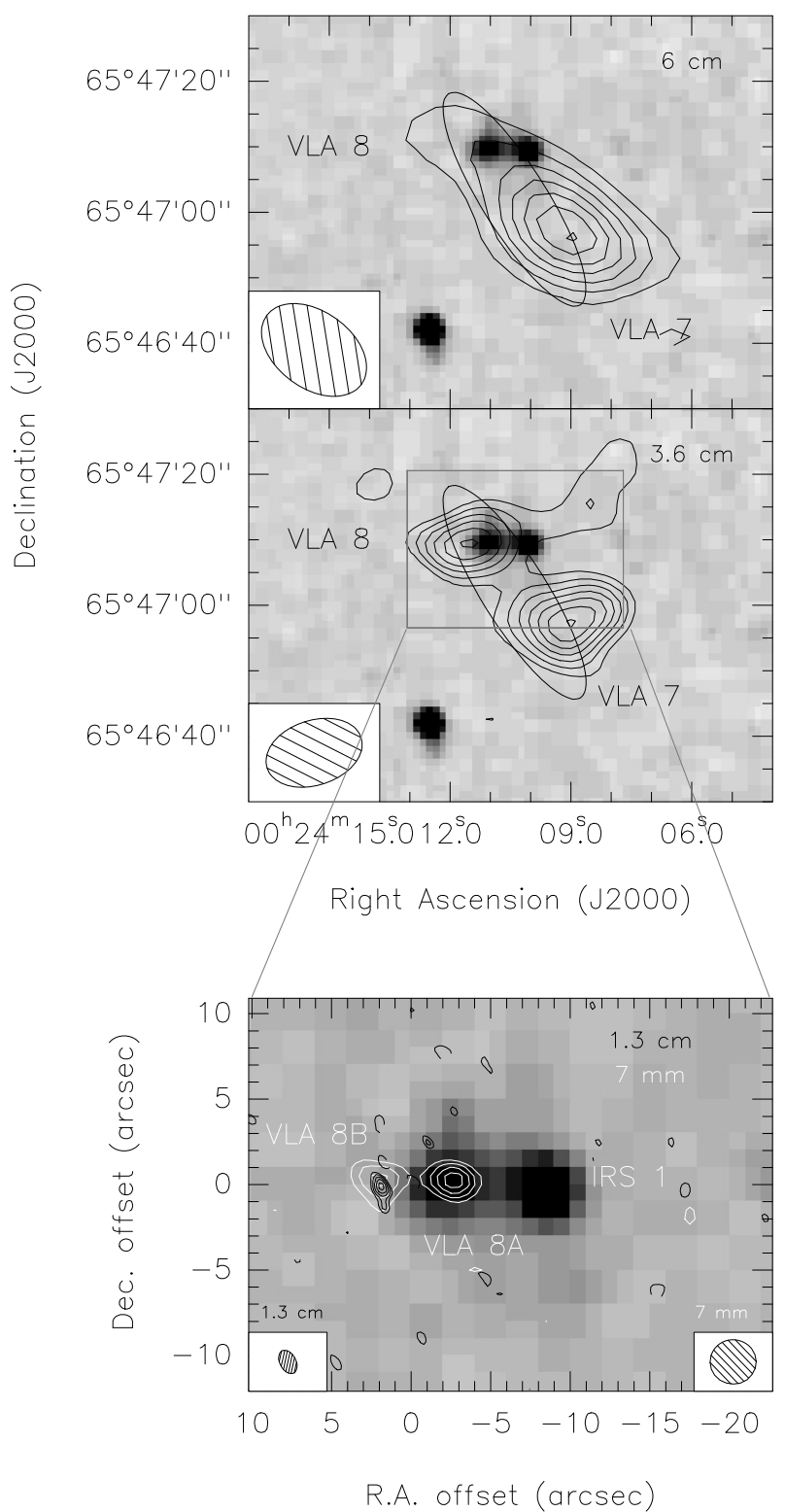

Fig. 3. Top: VLA contour map of the $6 \mathrm{~cm}$ continuum emission toward I00213. Contour levels are $-3,3,6,9,12,15,18$, and 21 times the rms of the map, $24 \mu \mathrm{Jy}$ beam $^{-1}$. Middle: VLA $3.6 \mathrm{~cm}$ continuum emission. Contour levels are $-3,3,4,5,6,7,8$ and 9 times the rms of the map, $18 \mu \mathrm{Jy}_{\text {beam }}{ }^{-1}$. The synthesized beams at $6 \mathrm{~cm}\left(17^{\prime \prime} .9 \times 11^{\prime \prime} .7\right.$, with $\left.\mathrm{PA}=54^{\circ}\right)$, and at $3.6 \mathrm{~cm}\left(15^{\prime \prime} .1 \times 99^{\prime} 9\right.$, with $\left.\mathrm{PA}=-72^{\circ}\right)$, are shown at the bottom left corner of each panel. Bottom: white: VLA $7 \mathrm{~mm}$ continuum emission, black: VLA $1.3 \mathrm{~cm}$ continuum emission. Contour levels at $7 \mathrm{~mm}$ are $-3,3,4,5$, and 6 times the rms of the map, $0.2 \mathrm{mJy}_{\text {beam }}{ }^{-1}$. Contour levels at $1.3 \mathrm{~cm}$ are $-3,3,4,5,6$, and 7 times the rms of the map, $0.15 \mathrm{mJy}_{\text {beam }}^{-1}$. The synthesized beams at $1.3 \mathrm{~cm}$ and $7 \mathrm{~mm}$ are shown at the bottom left and right corners of the image, respectively. In all panels the grey scale is the 2MASS $K_{\mathrm{s}}$-band image, and the position error of IRAS $00213+6530$ is indicated by the ellipse.

panel). While we did not detect $7 \mathrm{~mm}$ continuum emission toward VLA 7, the $7 \mathrm{~mm}$ emission of VLA 8 is resolved into two components, VLA $8 \mathrm{~A}$ and VLA $8 \mathrm{~B}$, separated by $\sim 5^{\prime \prime}$ ( $\sim 300 \mathrm{AU}$ at the distance of the source). At $1.3 \mathrm{~cm}$ we detected one source associated with VLA $8 \mathrm{~B}$, whose peak position coincides within $\sim 0$ '. 5 with the $7 \mathrm{~mm}$ peak (Fig. 3 bottom panel). The $1.3 \mathrm{~cm}$ source is elongated roughly in the northeast-southwest direction, and is spatially resolved only in one direction, with a deconvolved size of $1^{\prime \prime} .2(\sim 1020 \mathrm{AU})$, at PA $=21^{\circ}$. In Table 5 we show the position, peak intensity, and flux density of VLA 8A and VLA 8B at $1.3 \mathrm{~cm}$ and $7 \mathrm{~mm}$, as well as the spectral index between these wavelengths. In order to properly estimate the spectral index in the $1.3 \mathrm{~cm}-7 \mathrm{~mm}$ range, we applied a $u v$ taper function of $80 \mathrm{k} \lambda$ to obtain similar angular resolutions at both wavelengths (see Sect. 2.2). The resulting spectral indices are $>1.4$ and $0.2 \pm 0.6$ for VLA $8 \mathrm{~A}$ and VLA $8 \mathrm{~B}$, respectively.

As seen in Fig. 3 (bottom panel), VLA 8A coincides with the near infrared source 2MASS J00241110+6547095, whereas VLA 8B has no infrared emission associated with it. In addition, $\sim 6^{\prime \prime}$ ( $\sim 5100$ AU ) west of VLA 8A there is the near-infrared source IRS 1 with no detected $7 \mathrm{~mm}$ emission. At $7 \mathrm{~mm}$ both thermal dust emission and free-free emission can contribute to the total emission. Then, in order to estimate the mass of gas and dust from the $7 \mathrm{~mm}$ emission, we need first to know the flux density coming from thermal dust emission. Since the angular resolution achieved at $3.6 \mathrm{~cm}$ is not sufficient to resolve the two millimeter sources, we cannot estimate the contribution of free-free emission at $7 \mathrm{~mm}$ for each individual source. A first approach is to smooth the $7 \mathrm{~mm}$ emission to the angular resolution of the $3.6 \mathrm{~cm}$ data. The result is a single source with a flux density of $2.5 \pm 0.9 \mathrm{mJy}$, which is consistent with the sum of the flux densities of both millimeter sources. Extrapolating the flux obtained at $3.6 \mathrm{~cm}$ for VLA 8 to millimeter wavelengths with the spectral index obtained from the centimeter emission at $6 \mathrm{~cm}$ and $3.6 \mathrm{~cm}$ ( $\alpha=0.5$ ), we find that the expected free-free emission at $7 \mathrm{~mm}$ is $\sim 0.48 \mathrm{mJy}$ ( $19 \%$ of the $7 \mathrm{~mm}$ flux), indicating that the thermal dust component dominates at this wavelength. We note that the free-free contribution estimated at $7 \mathrm{~mm}$ can be considered as an upper limit because the free-free spectral index between $3.6 \mathrm{~cm}$ and $7 \mathrm{~mm}$ is expected to be flatter than between 6 and $3.6 \mathrm{~cm}$. However, the thermal dust contribution at $7 \mathrm{~mm}$ may be different for VLA 8A and VLA 8B. The fact that VLA 8A is not detected at $1.3 \mathrm{~cm}$ above a $4 \sigma$ level indicates that at $7 \mathrm{~mm}$ the emission is mainly due to thermal dust emission rather than free-free emission, whereas VLA 8B, which is associated with a $1.3 \mathrm{~cm}$ source, must have less thermal dust emission associated with it.

To estimate the mass, we assumed that the dust emission is optically thin, and used the opacity law of $\kappa_{v}=$ $0.01(v / 230 \mathrm{GHz})^{\beta} \mathrm{cm}^{2} \mathrm{~g}^{-1}$ (Ossenkopf \& Henning 1994), extrapolated to $7 \mathrm{~mm}$. We used a dust emissivity index $\beta=1.5$ (derived from the spectral energy distribution, see Sect. 4.2). The dust temperature is estimated by correcting the rotational temperature derived from $\mathrm{NH}_{3}(\sim 20 \mathrm{~K}$, see next sections) to kinetic temperature $(\sim 25 \mathrm{~K})$, following the expression of Tafalla et al. (2004). Using the fraction of the $7 \mathrm{~mm}$ flux density arising from thermal dust emission ( $\sim 2.1 \mathrm{mJy})$, the total mass derived for the two sources, VLA $8 \mathrm{~A}$ and VLA $8 \mathrm{~B}$, is $6.3 M_{\odot}$. It is worth noting that this mass is an upper limit since at $7 \mathrm{~mm}$ we are sensitive to spatial scales of $\sim 2500 \mathrm{AU}$, smaller than that achieved with the $\mathrm{NH}_{3}$ observations. Thus, the temperature should be higher than that estimated from the $\mathrm{NH}_{3}$ emission, and the resulting mass would be lower. The uncertainty in the mass is around a factor of 4 mainly due to uncertainties in the dust opacity and the dust emissivity index.

As interferometers are not sensitive to large-scale structures, we compared the $7 \mathrm{~mm}$ continuum emission with the $1.2 \mathrm{~mm}$ dust emission from the IRAM $30 \mathrm{~m}$ telescope. We followed the method described in Girart et al. (2000), which relates the $F W H M$ of the single-dish emission with the half-power $(u, v)$ radius, to estimate the magnitude of this effect at $7 \mathrm{~mm}$. By applying the relation of Girart et al. (2000), and adopting a FWHM 
Table 5. Parameters of sources detected at $1.3 \mathrm{~cm}$ and $7 \mathrm{~mm}$ in the IRAS $00213+6530$ region.

\begin{tabular}{|c|c|c|c|c|c|c|c|}
\hline \multirow[b]{2}{*}{ Source } & \multicolumn{2}{|c|}{ 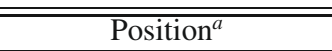 } & \multicolumn{2}{|c|}{$1.3 \mathrm{~cm}$} & \multicolumn{2}{|c|}{$7 \mathrm{~mm}$} & \multirow{2}{*}{$\begin{array}{c}\text { Spectral } \\
\text { Index }^{c} \\
1.3 \mathrm{~cm}-7 \mathrm{~mm}\end{array}$} \\
\hline & $\begin{array}{c}\alpha(\mathrm{J} 2000) \\
(\mathrm{h} \mathrm{m} \mathrm{s})\end{array}$ & $\begin{array}{c}\delta(\mathrm{J} 2000) \\
\left({ }^{\circ},{ }^{\prime \prime}\right)\end{array}$ & $\begin{array}{c}I_{v}{ }^{\text {peak }} \\
(\mathrm{mJy} \text { beam }\end{array}$ & $\begin{array}{c}S_{v} \\
(\mathrm{mJy})\end{array}$ & $\begin{array}{c}I_{v}{ }^{\text {peak }} \\
(\mathrm{mJy} \text { beam } \\
\end{array}$ & $\begin{array}{c}S_{v} \\
(\mathrm{mJy})\end{array}$ & \\
\hline $\begin{array}{l}\text { VLA 8A } \\
\text { VLA 8B }\end{array}$ & $\begin{array}{lll}00 & 24 & 11.01 \\
00 & 24 & 11.76\end{array}$ & $\begin{array}{l}654709.9 \\
654709.8\end{array}$ & $1.09 \pm 0.13$ & $\begin{array}{c}<0.6^{b} \\
1.25 \pm 0.25\end{array}$ & $\begin{array}{l}1.22 \pm 0.26 \\
1.12 \pm 0.38\end{array}$ & $\begin{array}{l}1.57 \pm 0.54 \\
1.43 \pm 0.79\end{array}$ & $\begin{array}{c}>1.4 \\
0.2+0.6\end{array}$ \\
\hline
\end{tabular}

${ }^{a}$ From the 7 mm map. ${ }^{b} 4 \sigma$ upper limit. ${ }^{c}$ Estimated from the $1.3 \mathrm{~cm}$ flux density obtained with a uv-taper function of $80 \mathrm{k} \lambda$ (see main text).

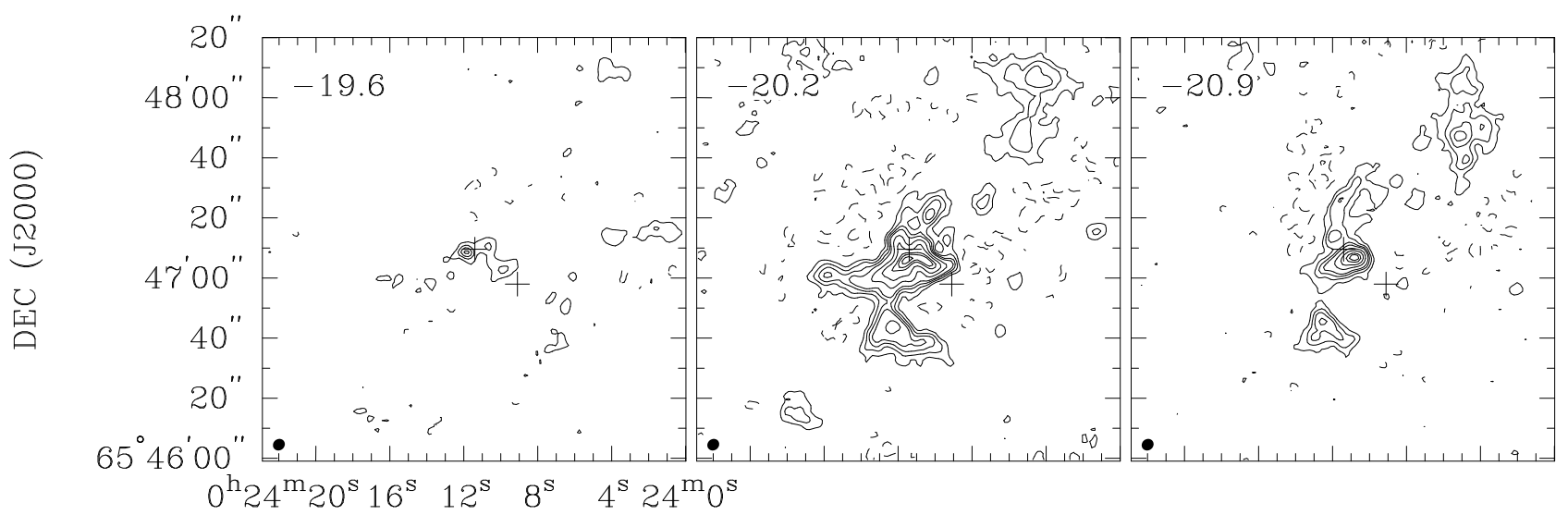

RA (J2000)

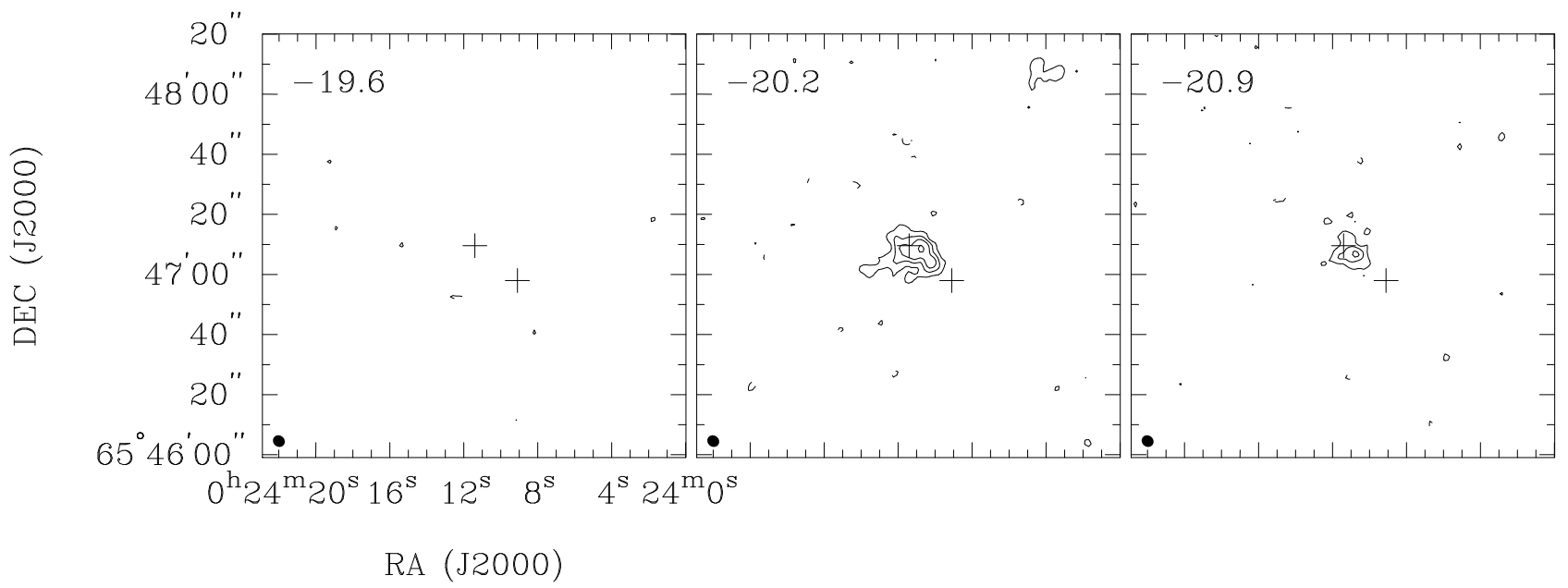

Fig. 4. Top panel: VLA channel maps of the $\mathrm{NH}_{3}(1,1)$ main line. Contours levels are $-6,-3,3,6,9,12,18,24,30,33$, and 36 times the rms noise of the map, $1.1 \mathrm{mJy}_{\text {beam }}{ }^{-1}$. Bottom panel: VLA channel maps of the $\mathrm{NH}_{3}(2,2)$ main line. Contour levels are $-3,3,6,9$, and 12 times the rms noise of the map, $1.1 \mathrm{mJy}^{\text {beam }}{ }^{-1}$. In both panels the synthesized beam is shown in the bottom left corner. The positions of VLA 7 and VLA 8 are indicated by crosses.

for MM1 of $\theta_{F W H M} \simeq\left(13^{\prime \prime} \times 10^{\prime \prime}\right)^{1 / 2}=11^{\prime \prime}$ the half-power $(u, v)$ radius of the $1.2 \mathrm{~mm}$ dust emission MM1 becomes $r \simeq 8 \mathrm{k} \lambda$. Given the shortest baseline of the VLA in the D configuration, which is $2.5 \mathrm{k} \lambda<8 \mathrm{k} \lambda$, and the size of the observed emission one can estimate the fraction of correlated flux detected by the interferometer. For a source of $11^{\prime \prime}$, this corresponds to $94 \%$ for our VLA configuration at $7 \mathrm{~mm}$, indicating that at $7 \mathrm{~mm}$ we are not filtering out too much dust emission.

\section{3. $\mathrm{NH}_{3}(1,1)$ and $(2,2)$}

$\mathrm{NH}_{3}(1,1)$ and $\mathrm{NH}_{3}(2,2)$ emission is detected in individual velocity channels from -19.6 to $-20.9 \mathrm{~km} \mathrm{~s}^{-1}$ and from -20.2 to $-20.9 \mathrm{~km} \mathrm{~s}^{-1}$, respectively. We also detected the inner satellite lines as well as one of the outer satellite lines of the $(1,1)$ transition, and we marginally detected one of the inner satellite lines of the $\mathrm{NH}_{3}(2,2)$. Figure 4 shows the velocity channel maps of the $\mathrm{NH}_{3}(1,1)$ and $(2,2)$ main line emission, and in Fig. 5 we show the $\mathrm{NH}_{3}(1,1)$ and $(2,2)$ spectra, not corrected for the primary beam response, at some selected positions. In Table 6 we show the line parameters toward these positions obtained from a Gaussian fit to the $\mathrm{NH}_{3}(1,1)$ main and inner satellites lines, as well as the $\mathrm{NH}_{3}(2,2)$ line. We additionally show the ratio of the main line to the inner satellites, which gives an indication of the line optical depth. The values obtained for the optical depth are in the range $\tau_{\mathrm{m}} \sim 2-3$. In Table 7 we present the line parameters resulting from the hyperfine fit to the $\mathrm{NH}_{3}(1,1)$ line toward the same positions. Given our spectral resolution, $\Delta v=0.6 \mathrm{~km} \mathrm{~s}^{-1}$, the intrinsic line width has been obtained by selecting the value 


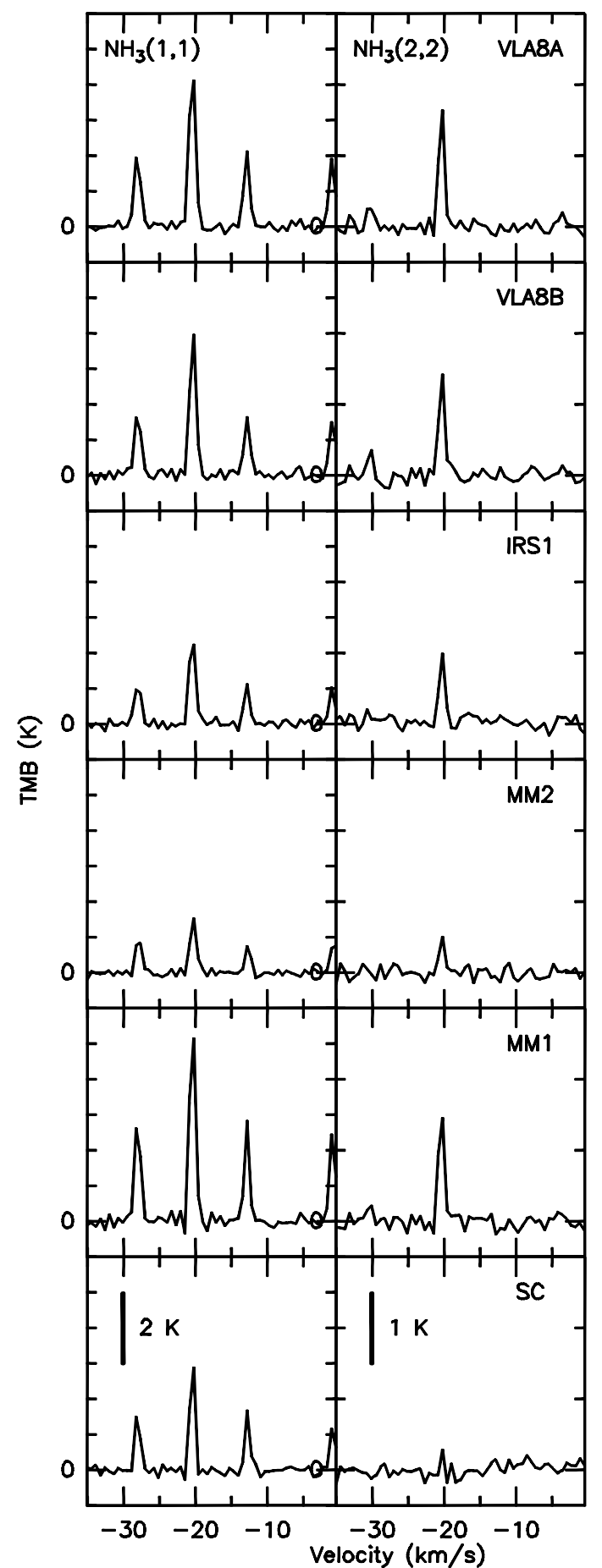

Fig. 5. Spectra toward six positions of the IRAS $00213+6530$ region for $\mathrm{NH}_{3}(1,1)$ (left) and $\mathrm{NH}_{3}(2,2)$ (right), averaged over one beam. The six positions, which are labeled on the right panel of each row, are, from top to bottom, VLA 8A, VLA 8B, IRS 1, MM2 (peak of the northwestern cloud), MM1 (peak of the central cloud), and SC (peak of the southern cloud). The vertical scale for each transition is indicated in the bottom row.

that minimize the hyperfine fit residual. The optical depths derived from the magnetic hyperfine fit are systematically higher but compatible with the values obtained from the Gaussian fit (see Appendix for further details).

In Fig. 6 we present the zero-order moment map of the $\mathrm{NH}_{3}(1,1)$ and $(2,2)$ emission (integrated intensity including only the main line). While the $\mathrm{NH}_{3}(1,1)$ map shows significant

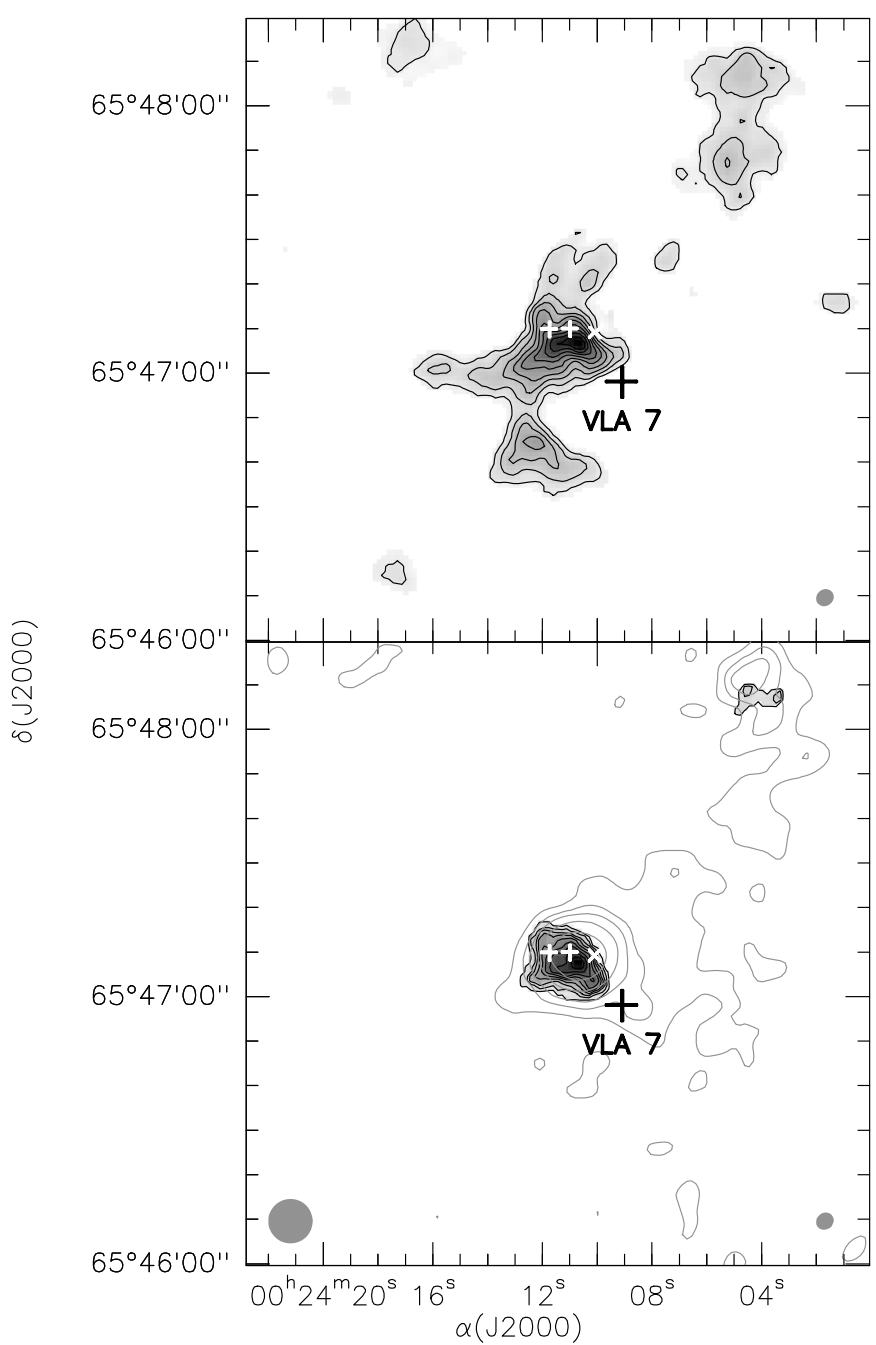

Fig. 6. Top panel: zero-order moment of the $\mathrm{NH}_{3}(1,1)$ main line emission. Bottom panel: zero-order moment map of the $\mathrm{NH}_{3}(2,2)$ main line emission (black contours). Grey contours: $1.2 \mathrm{~mm}$ continuum emission. Grey contour levels are 3, 6, 9, 12, 25, and 35 times the rms of the map, $3.8 \mathrm{mJy} \mathrm{beam}^{-1}$. In both panels, black contours start at $10 \%$, increasing in steps of $10 \%$ of the peak intensity, $0.0508 \mathrm{Jy} \mathrm{beam}^{-1} \mathrm{~km} \mathrm{~s}^{-1}$ and $0.0151 \mathrm{Jy} \mathrm{beam}^{-1} \mathrm{~km} \mathrm{~s}^{-1}$ for $\mathrm{NH}_{3}(1,1)$ and $(2,2)$, respectively. White crosses mark the position of the two millimeter sources, VLA 8A and VLA $8 \mathrm{~B}$, and the white tilted cross marks the position of the infrared source IRS 1 . The synthesized beams for each transition are shown at the bottom right corner, and the synthesized beam of the $1.2 \mathrm{~mm}$ continuum data is shown in the bottom left corner of the bottom panel.

extended structure, the emission of the $\mathrm{NH}_{3}(2,2)$ is compact around the position of the centimeter/millimeter sources, We also detected faint $\mathrm{NH}_{3}(2,2)$ emission toward the northern peak of MM2. The overall structure of the $\mathrm{NH}_{3}(1,1)$ emission consists of three clouds. The central $\mathrm{NH}_{3}$ cloud coincides with the dust condensation MM1. Around 22" south of MM1 there is another cloud detected only in $\mathrm{NH}_{3}$ (hereafter southern cloud). Moreover, the $\mathrm{NH}_{3}$ cloud located to the northwest of MM1 is associated with the dust condensation MM2. Note that, because of the small size of the VLA primary beam at $1.3 \mathrm{~cm}$, the sensitivity toward MM2 is two times lower than toward the center of the field. Then, the dense gas emission traced by the $\mathrm{NH}_{3}$ molecule roughly follows the $1.2 \mathrm{~mm}$ dust continuum emission, except in the southern cloud in which we did not detect dust emission at all (see Fig. 6). While the $\mathrm{NH}_{3}$ cloud MM1 engulfs the centimeter source VLA 8 (i.e., IRS 1, VLA 8A, 
Table 6. $\mathrm{NH}_{3}$ line parameters obtained from the Gaussian fits to the $\mathrm{NH}_{3}(1,1)$ and $(2,2)$.

\begin{tabular}{|c|c|c|c|c|c|c|c|c|c|}
\hline \multirow[b]{2}{*}{ Source } & \multicolumn{2}{|c|}{ Position } & \multirow[b]{2}{*}{$\begin{array}{c}\text { Transition } \\
(J, K)\end{array}$} & \multicolumn{3}{|c|}{ Main line } & \multicolumn{2}{|c|}{ Inner satellites } & \multirow[b]{2}{*}{$\frac{T_{\mathrm{MB}}(m)}{T_{\mathrm{MB}}(i s)}$} \\
\hline & $\begin{array}{c}\alpha(\mathrm{J} 2000) \\
(\mathrm{h} \mathrm{m} \mathrm{s})\end{array}$ & $\begin{array}{c}\delta(\mathrm{J} 2000) \\
\left({ }^{\circ} \prime^{\prime \prime \prime}\right)\end{array}$ & & $\begin{array}{c}v_{\mathrm{LSR}} \\
\left(\mathrm{km} \mathrm{s}^{-1}\right)\end{array}$ & $\begin{array}{c}T_{\mathrm{MB}} \\
(\mathrm{K})\end{array}$ & $\begin{array}{c}\Delta v \\
\left(\mathrm{~km} \mathrm{~s}^{-1}\right)\end{array}$ & $\begin{array}{l}T_{\mathrm{MB}} \\
(\mathrm{K})\end{array}$ & $\begin{array}{c}\Delta v \\
\left(\mathrm{~km} \mathrm{~s}^{-1}\right)\end{array}$ & \\
\hline \multirow{2}{*}{ VLA 8A } & 002411.01 & +654709.9 & $(1,1)$ & $-19.98 \pm 0.01$ & $3.9 \pm 0.2$ & $1.19 \pm 0.02$ & $1.8 \pm 0.2$ & $1.14 \pm 0.04$ & 2.2 \\
\hline & & & $(2,2)$ & $-19.92 \pm 0.03$ & $1.8 \pm 0.1$ & $0.83 \pm 0.07$ & & & \\
\hline \multirow[t]{2}{*}{ VLA 8B } & 002411.76 & +654709.8 & $(1,1)$ & $-19.89 \pm 0.01$ & $3.6 \pm 0.2$ & $1.16 \pm 0.02$ & $1.5 \pm 0.2$ & $1.18 \pm 0.06$ & 2.4 \\
\hline & & & $(2,2)$ & $-19.88 \pm 0.04$ & $1.5 \pm 0.1$ & $0.91 \pm 0.08$ & & & \\
\hline \multirow[t]{2}{*}{$\overline{\mathrm{IRS}} 1$} & 002410.10 & +654709.1 & $(1,1)$ & $-19.97 \pm 0.02$ & $2.2 \pm 0.1$ & $1.19 \pm 0.03$ & $1.0 \pm 0.1$ & $1.11 \pm 0.07$ & 2.2 \\
\hline & & & $(2,2)$ & $-19.83 \pm 0.05$ & $1.0 \pm 0.1$ & $0.92 \pm 0.08$ & & & \\
\hline \multirow[t]{2}{*}{ MM2 } & 002412.30 & +654645.0 & $(1,1)$ & $-19.84 \pm 0.02$ & $1.4 \pm 0.1$ & $1.19 \pm 0.05$ & $0.9 \pm 0.1$ & $1.06 \pm 0.05$ & 1.6 \\
\hline & & & $(2,2)$ & $-19.85 \pm 0.08$ & $0.5 \pm 0.1$ & $0.88 \pm 0.17$ & & & \\
\hline \multirow[t]{2}{*}{ MM1 } & 002410.80 & +654706.0 & $(1,1)$ & $-19.94 \pm 0.01$ & $4.8 \pm 0.2$ & $1.10 \pm 0.03$ & $2.5 \pm 0.1$ & $1.02 \pm 0.04$ & 1.9 \\
\hline & & & $(2,2)$ & $-19.96 \pm 0.04$ & $1.8 \pm 0.1$ & $0.83 \pm 0.10$ & & & \\
\hline$\overline{\mathrm{SC}}$ & 002405.30 & +654747.0 & $(1,1)$ & $-19.95 \pm 0.01$ & $2.7 \pm 0.1$ & $0.97 \pm 0.02$ & $1.5 \pm 0.1$ & $0.95 \pm 0.05$ & 1.8 \\
\hline
\end{tabular}

Table 7. $\mathrm{NH}_{3}$ line parameters from the fits to the $\mathrm{NH}_{3}(1,1)$ magnetic hyperfine components.

\begin{tabular}{lccrc}
\hline \hline Source & $\begin{array}{c}v_{\mathrm{LSR}} \\
\left(\mathrm{km} \mathrm{s}^{-1}\right)\end{array}$ & $\begin{array}{c}\Delta v^{a} \\
\left(\mathrm{~km} \mathrm{~s}^{-1}\right)\end{array}$ & $\begin{array}{c}A \\
(\mathrm{~K})\end{array}$ & $\tau_{\mathrm{m}}^{b}$ \\
\hline VLA 8A & $-19.99 \pm 0.01$ & $0.8 \pm 0.1$ & $11.04 \pm 0.32$ & $2.71 \pm 0.13$ \\
VLA 8B & $-19.92 \pm 0.01$ & $0.9 \pm 0.1$ & $8.41 \pm 0.35$ & $2.12 \pm 0.16$ \\
IRS 1 & $-19.92 \pm 0.01$ & $0.9 \pm 0.1$ & $8.39 \pm 0.34$ & $2.11 \pm 0.15$ \\
MM2 & $-19.85 \pm 0.01$ & $0.7 \pm 0.1$ & $6.60 \pm 0.34$ & $4.40 \pm 0.37$ \\
MM1 & $-19.96 \pm 0.01$ & $0.7 \pm 0.1$ & $16.34 \pm 0.54$ & $3.46 \pm 0.17$ \\
SC & $-19.96 \pm 0.01$ & $0.6 \pm 0.1$ & $10.08 \pm 0.49$ & $3.96 \pm 0.27$ \\
\hline
\end{tabular}

${ }^{a}$ Intrinsic line width $(F W H M)$ of the magnetic hyperfine component. ${ }^{b} A=f\left(J_{v}\left(T_{\mathrm{ex}}\right)-J_{v}\left(T_{\mathrm{bg}}\right)\right)$, where $f$ is the filling factor, $T_{\mathrm{ex}}$ is the excitation temperature of the transition, $T_{\mathrm{bg}}$ is the background radiation temperature, and $J_{v}(T)$ is the intensity in units of temperature, $J_{v}(T)=(h v / k) /(\exp (h v / k T)-1) .{ }^{c}$ Optical depth of the main line obtained from the fit.

and VLA 8B), no $\mathrm{NH}_{3}$ emission is seen toward the position of VLA 7, which falls close to the edge of the $\mathrm{NH}_{3}$ emission. It is important to note that there is a near-infrared source detected in the 2MASS bands, 2MASS J00241251+6546418, spatially coinciding with the southern $\mathrm{NH}_{3}$ cloud, which probably is not associated with the dense gas (see Sect. 5.2 for a complete explanation).

In Fig. 7 (top) we show the first-order moment (intensity weighted mean $\left.v_{\mathrm{LSR}}\right)$ of the $\mathrm{NH}_{3}(1,1)$ main line emission. As can be seen in this figure, the velocity along the central ammonia cloud MM1 shows only small variations, and no significant velocity gradients are found between MM1 and the southern cloud. The millimeter source VLA $8 \mathrm{~B}$ is redshifted by $\sim 0.3 \mathrm{~km} \mathrm{~s}^{-1}$ with respect to VLA 8A. In addition toward the western and eastern edges of MM1 the $\mathrm{NH}_{3}$ emission is redshifted by $\sim 0.4 \mathrm{~km} \mathrm{~s}^{-1}$. Toward MM2 there is a small velocity gradient in the north-south direction of $\sim 0.6 \mathrm{~km} \mathrm{~s}^{-1}$ along a region of $\sim 30^{\prime \prime}$.

The map of the second-order moment (intensity weighted velocity dispersion) of the $\mathrm{NH}_{3}(1,1)$ main line emission is shown in Fig. 7 (bottom). The typical value found for the velocity dispersion in the southern cloud is $\sim 0.25-0.3 \mathrm{~km} \mathrm{~s}^{-1}$, which corresponds for a Gaussian line profile to a full width at half maximum $(F W H M)$ of $0.6 \mathrm{~km} \mathrm{~s}^{-1}$, similar to the instrumental resolution. In contrast, in the $\mathrm{NH}_{3}$ cloud MM1 there is evidence of line broadening toward the three embedded sources (forming an arc-shaped structure), with values up to $0.4-0.5 \mathrm{~km} \mathrm{~s}^{-1}$, corresponding to line widths of $0.7-1 \mathrm{~km} \mathrm{~s}^{-1}$, corrected for instrumental resolution, significantly higher than the expected

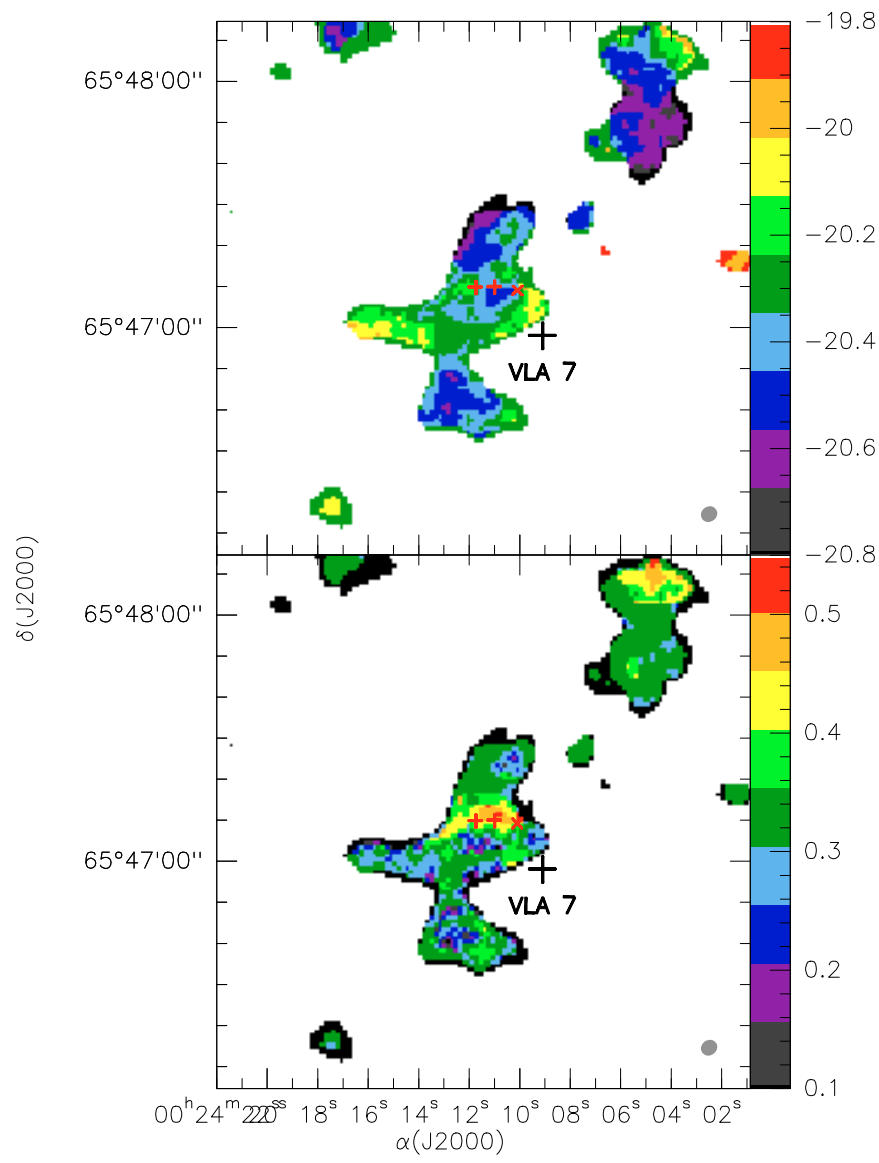

Fig. 7. Top panel: first-order moment map of the $\mathrm{NH}_{3}(1,1)$ main line emission. Bottom panel: second-order moment map of the $\mathrm{NH}_{3}(1,1)$ main line emission. Symbols are the same as in Fig. 6. Color wedge scales are $\mathrm{km} \mathrm{s}^{-1}$. The synthesized beam is shown at the bottom right corner of the images. Note that the second-order moment gives the velocity dispersion, and must be multiplied by the factor $2 \sqrt{2 \ln 2} \simeq 2.35$ to convert to full width at half maximum.

thermal line width $\sim 0.23 \mathrm{~km} \mathrm{~s}^{-1}$ (estimated for a kinetic temperature of $\sim 20 \mathrm{~K}$ ), indicative of a significant contribution from non-thermal processes. We found that the typical value of velocity dispersion in the $\mathrm{NH}_{3}$ cloud MM2 is $\sim 0.3-0.35 \mathrm{~km} \mathrm{~s}^{-1}$, corresponding to line widths of $0.4-0.5 \mathrm{~km} \mathrm{~s}^{-1}$, corrected for instrumental resolution, and rises up to $0.45 \mathrm{~km} \mathrm{~s}^{-1}$ (line width of $0.9 \mathrm{~km} \mathrm{~s}^{-1}$, corrected for instrumental resolution) toward the northern peak of MM2. These values are slightly higher than 
Table 8. Summary of $\mathrm{H}_{2} \mathrm{O}$ maser observations toward IRAS 00213+6530.

\begin{tabular}{|c|c|c|c|c|c|c|c|}
\hline Reference & $\begin{array}{c}\text { Observation } \\
\text { date }\end{array}$ & Telescope & Beam & $\begin{array}{c}\text { Positional } \\
\text { accuracy }\end{array}$ & Detection & $\begin{array}{c}\mathrm{rms} \\
\left(\mathrm{Jy} \mathrm{beam}^{-1}\right)\end{array}$ & $\begin{array}{c}I_{\text {peak }} \\
\left(\text { Jy beam }^{-1}\right)\end{array}$ \\
\hline Felli et al. (1992) & 1991 Jan. 18 & $32 \mathrm{~m}$ Medicina & 1.9 & $15^{\prime \prime}$ & no & 1.1 & $<5.5$ \\
\hline Anglada et al. (1997) & 1990 Feb. & 37 m Haystack & 1.4 & $15^{\prime \prime}$ & no & 1.6 & $<4.8$ \\
\hline Han et al. (1998) & 1993 Nov. 21 & 13.7 m Purple Mount & 4.2 & $20^{\prime \prime}$ & yes $^{a}$ & 9.1 & 38.8 \\
\hline this work & 2006 Dec. 2 & VLA & $2{ }^{\prime \prime} 1$ & $<1^{\prime \prime}$ & no & 0.003 & $<0.015$ \\
\hline this work & 2008 Apr. 18 & NASA $70 \mathrm{~m}$ & $40^{\prime \prime}$ & $10^{\prime \prime}$ & yes $^{b}$ & 0.03 & 0.111 \\
\hline this work & 2008 Jun. 19 & NASA $70 \mathrm{~m}$ & $40^{\prime \prime}$ & $10^{\prime \prime}$ & no & 0.04 & $<0.21$ \\
\hline this work & 2008 Sep. 23 & NASA $70 \mathrm{~m}$ & $40^{\prime \prime}$ & $10^{\prime \prime}$ & no & 0.06 & $<0.28$ \\
\hline
\end{tabular}

${ }^{a}$ Detection at $-0.7 \mathrm{~km} \mathrm{~s}^{-1} \cdot{ }^{b}$ Marginal detection at $-15 \mathrm{~km} \mathrm{~s}^{-1}$.

those found in the southern cloud, suggesting that the gas is being perturbed in the $\mathrm{NH}_{3}$ cloud MM2.

Finally, we compared the VLA $\mathrm{NH}_{3}(1,1)$ emission with the single-dish $\mathrm{NH}_{3}(1,1)$ emission of Sepúlveda (2001). From the VLA $\mathrm{NH}_{3}$ observations we estimated a peak intensity of $27.8 \mathrm{mJy}$ beam $^{-1}$, which corresponds to a main beam brightness temperature $T_{\mathrm{MB}} \simeq 4 \mathrm{~K}$. The largest angular scale detectable by the VLA at $1.3 \mathrm{~cm}$ in the D configuration is around $60^{\prime \prime}$, and the size of the largest features detected by us with the VLA is $\sim 30^{\prime \prime}$. From the size of the $\mathrm{NH}_{3}$ emission we can estimate the dilution effect when observed this emission with a single-dish telescope of 1.4. The dilution effect would decrease the main beam brightness temperature, $T_{\mathrm{MB}}$, by a factor of 7.8 , consistent with the $T_{\mathrm{MB}}$ measured by Sepúlveda (2001), indicating that the fraction of emission filtered out by the interferometer must be small or negligible.

\section{4. $\mathrm{H}_{2} \mathrm{O}$ maser emission}

In Table 8 we compiled the $\mathrm{H}_{2} \mathrm{O}$ maser observations carried out toward I00213 up to date, including our observations with the VLA and NASA $70 \mathrm{~m}$. The $\mathrm{H}_{2} \mathrm{O}$ maser emission was clearly detected only in 1993 by Han et al. (1998), using the $13.7 \mathrm{~m}$ radio telescope of Purple Mountain Observatory, with a peak intensity of $38.8 \mathrm{Jy}$. We did not detect $\mathrm{H}_{2} \mathrm{O}$ maser emission toward I00213, except in the NASA $70 \mathrm{~m}$ observations on 2008 April 18, where we marginally detected emission at a $3 \sigma$ level of $0.1 \mathrm{Jy} \mathrm{beam}^{-1}$ (see Fig. 8). The integrated intensity was $\sim 0.49 \mathrm{Jy} \mathrm{beam}^{-1} \mathrm{~km} \mathrm{~s}^{-1}$, and the velocity of the feature was $-15.2 \mathrm{~km} \mathrm{~s}^{-1}$, offset by $\sim 5 \mathrm{~km} \mathrm{~s}^{-1}$ from the velocity of the cloud, $\sim-20 \mathrm{~km} \mathrm{~s}^{-1}$.

The characterization of $\mathrm{H}_{2} \mathrm{O}$ maser emission is difficult because of its high temporal variability, and thus it is possible that there was no maser during the epochs of observation (2006 Dec and 2008) with the exception of the marginal detection during 2008 April. However, it is not clear whether the $\mathrm{H}_{2} \mathrm{O}$ maser detected by Han et al. (1998) is associated with the I00213 region. On one hand, the maser emission was detected at $v_{\mathrm{LSR}}=$ $-0.7 \mathrm{~km} \mathrm{~s}^{-1}, 19.2 \mathrm{~km} \mathrm{~s}^{-1}$ offset from the systemic velocity of the cloud studied in this work. On the other hand, the beam of the $13.7 \mathrm{~m}$ radio telescope at this wavelength is 4.2 , with a pointing accuracy of $20^{\prime \prime}$, making it difficult to ascertain whether the maser is associated with the IRAS source. In addition, other attempts to detect $\mathrm{H}_{2} \mathrm{O}$ maser emission toward $\mathrm{I} 00213$ have failed (Felli et al. 1992; C. Codella observed the source between 1989 and 1999 using the $32 \mathrm{~m}$ Medicina telescope but did not detect it; private communication). Thus, the maser activity of I00213 seems to be currently in a rather quiescent state.

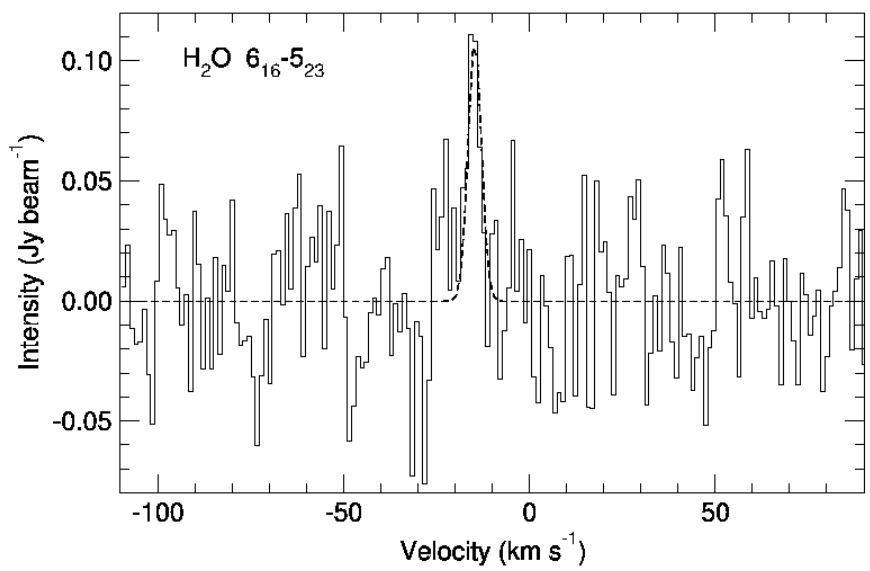

Fig. 8. Spectrum of the $\mathrm{H}_{2} \mathrm{O}$ maser observed with the NASA $70 \mathrm{~m}$ telescope on 2008 April 18. The spectrum was smoothed to a spectral resolution of $1.13 \mathrm{~km} \mathrm{~s}^{-1}$. The dashed line is a Gaussian fit to the spectrum.

Table 9. Parameters of the NASA $70 \mathrm{~m}$ CCS line emission toward I00213.

\begin{tabular}{lccc}
\hline \hline Line & $\begin{array}{c}v_{\mathrm{LSR}} \\
\left(\mathrm{km} \mathrm{s}^{-1}\right)\end{array}$ & $\begin{array}{c}\Delta v \\
\left(\mathrm{~km} \mathrm{~s}^{-1}\right)\end{array}$ & $\begin{array}{c}T_{\mathrm{MB}} \\
(\mathrm{K})\end{array}$ \\
\hline $\mathrm{CCS} J_{N}=2_{1}-1_{0}$ & $-20.6 \pm 0.2$ & $1.3 \pm 0.5$ & $0.22 \pm 0.05$ \\
\hline
\end{tabular}

\subsection{CCS emission}

In Table 9 we show the parameters obtained from a Gaussian fit of the CCS line detected with the NASA $70 \mathrm{~m}$, after combining the data of the two days observed, and we show its spectrum in Fig. 9. The line is centered around $-20 \mathrm{~km} \mathrm{~s}^{-1}$, the same velocity as $\mathrm{NH}_{3}$, and its line width $(F W H M)$ is $\sim 1.3 \mathrm{~km} \mathrm{~s}^{-1}$, larger than the expected thermal line width for a kinetic temperature of $\sim 20 \mathrm{~K}$ (estimated from $\mathrm{NH}_{3}$ ), which is of $\sim 0.13 \mathrm{~km} \mathrm{~s}^{-1}$. This could be indicative of the CCS line width having a strong contribution from non-thermal processes, such as turbulence injected by the passage of an outflow and/or global systematic motions. The measured line width is higher than the largest line width measured by de Gregorio-Monsalvo et al. (2006) toward a sample of low-mass YSOs. A high angular resolution study of de Gregorio-Monsalvo et al. (2005) in CCS shows that this molecule is possibly enhanced via shocked-induced chemistry, and has a velocity gradient in the same direction of the outflow.

\section{Analysis}

\subsection{Rotational temperature and column density maps}

We computed maps of the rotational temperature and column density of $\mathrm{NH}_{3}$. To do this, we extracted the $\mathrm{NH}_{3}(1,1)$ and $(2,2)$ 


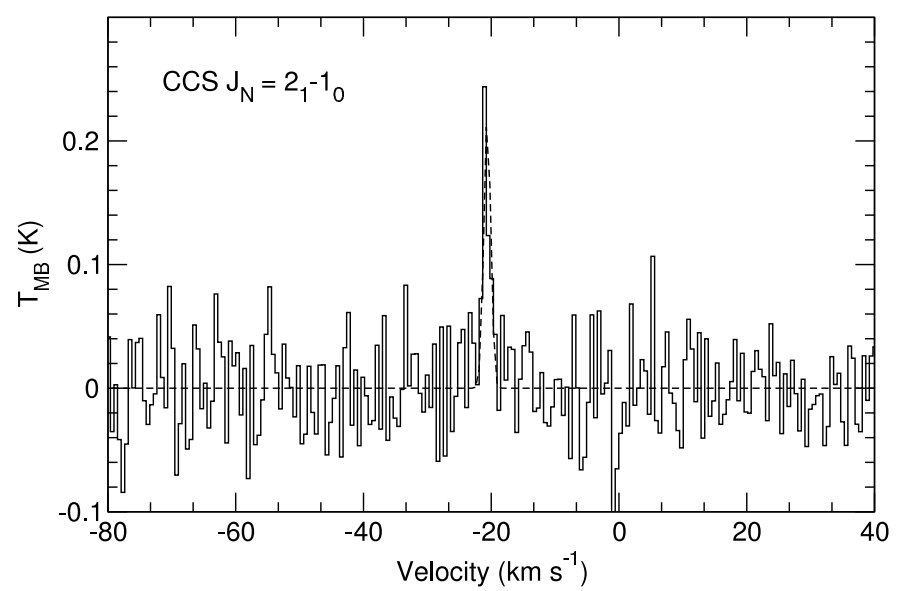

Fig. 9. Spectrum of the CCS $J_{N}=2_{1}-1_{0}$ transition observed with the NASA $70 \mathrm{~m}$ telescope toward I00213. The dashed line is a Gaussian fit to the spectrum.

spectra on a grid of points separated by $1^{\prime \prime}$ in the $\mathrm{NH}_{3}$ cloud MM1. We fitted the hyperfine structure of the $\mathrm{NH}_{3}(1,1)$ and a single Gaussian to the $\mathrm{NH}_{3}(2,2)$ for each spectrum. For the $\mathrm{NH}_{3}(1,1)$ transition we fitted only the spectra with an intensity greater than $5 \sigma$ in order to ensure the detection of the satellite lines, whereas for the $\mathrm{NH}_{3}(2,2)$ we fitted the spectra with an intensity greater than $4 \sigma$.

From the results of the fits of $\mathrm{NH}_{3}(1,1)$ and $\mathrm{NH}_{3}(2,2)$ we computed the rotational temperature $\left(T_{\text {rot }}\right)$ and $\mathrm{NH}_{3}$ column density maps following the standard procedures (Ho \& Townes 1983; Harju et al. 1993; Sepúlveda 1993; Anglada et al. 1995, see Appendix for a complete description of their derivation). This analysis assumes implicitly that the physical conditions of the gas are homogeneous along the line-of-sight, i.e., the excitation and the rotational temperature are constant along the lineof sight. Since gradients are probably present along the lineof-sight (see Sect. 4.2), the values obtained from this analysis should be considered as an "average" along the line of sight.

The map of the "average" rotational temperature obtained is shown in Fig. 10a. Interestingly, to the north of the millimeter sources VLA 8A and VLA 8B, there is a temperature enhancement, reaching a maximum value of $20 \pm 2 \mathrm{~K}$. Toward the millimeter sources the "average" rotational temperature is around $16 \mathrm{~K}$, and it decreases toward the south. In addition, at the western edge of the $\mathrm{NH}_{3}$ cloud MM1 and toward the infrared source IRS 1 there is a temperature enhancement. The "average" rotational temperature obtained at the position of IRS 1 is $\sim 15 \pm 1 \mathrm{~K}$, which is consistent with the expected association of this source with the high density gas. We also found a local maximum of temperature around $8^{\prime \prime}$ to the southwest of VLA 8A.

Figure 10b shows the resulting column density map for $\mathrm{NH}_{3}$, obtained after correction for the primary beam response. The highest values of the $\mathrm{NH}_{3}$ column density, $2.5 \times 10^{15} \mathrm{~cm}^{-2}$, are found to the south of the millimeter sources, where the rotational temperature shows the smallest values. Toward the two millimeter sources, VLA $8 \mathrm{~A}$ and VLA $8 \mathrm{~B}$, the $\mathrm{NH}_{3}$ column density is $\sim 8 \times 10^{14} \mathrm{~cm}^{-2}$.

\subsection{Radial intensity profile and Spectral Energy Distribution}

In order to study the spatial structure of the dusty condensation MM1 detected at $1.2 \mathrm{~mm}$, we computed the circularly averaged radial intensity profile, in rings of $3^{\prime \prime}$ width, as a function

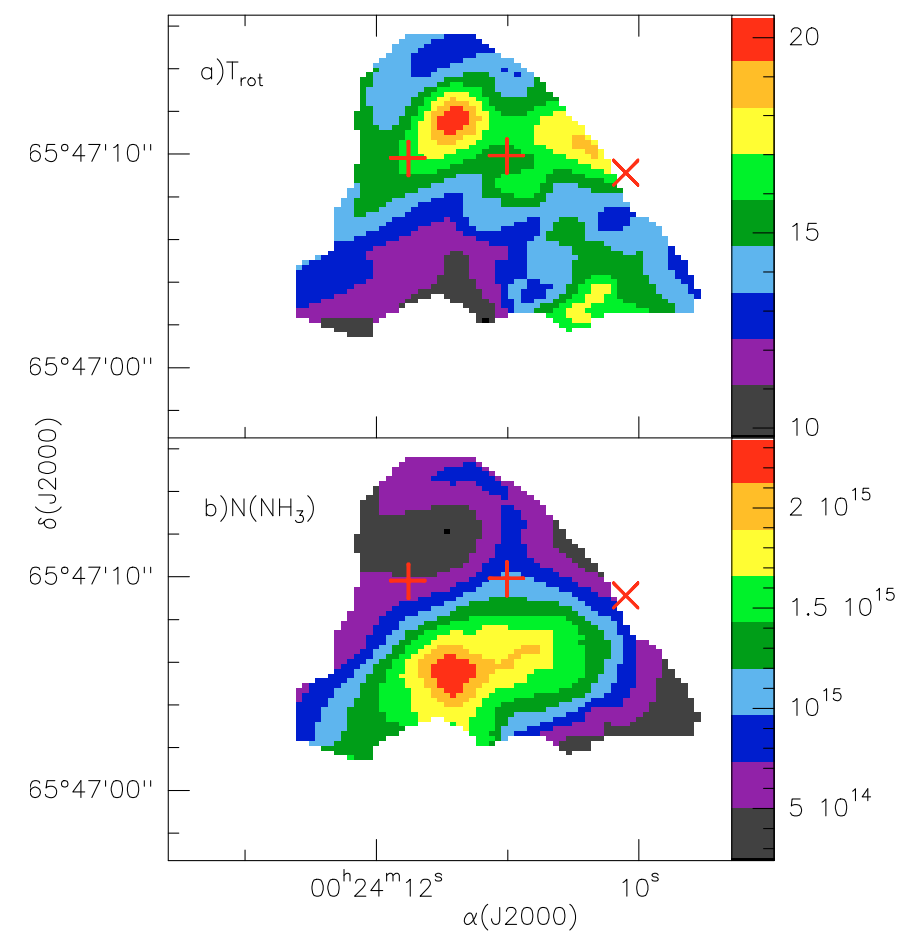

Fig. 10. a) "Average" Rotational temperature map from $\mathrm{NH}_{3}(1,1)$ and $\mathrm{NH}_{3}(2,2)$ toward MM1 (see text). Scale units are in $\mathrm{K}$. b) $\mathrm{NH}_{3}$ column density map. Scale units are in $\mathrm{cm}^{-2}$. In both panels red crosses mark the position of the two millimeter sources, VLA 8A and VLA 8B, and the red tilted cross marks the position of IRS 1. Note that VLA 7 lies outside the limits of this plot.

Table 10. Parameters of the envelope model used to fit the radial intensity profile of the $1.2 \mathrm{~mm}$ continuum emission and the SED of MM1.

\begin{tabular}{|c|c|}
\hline Parameter & Value \\
\hline Dust emissivity index $\beta$ & 1.5 \\
\hline$T$ power-law index & $0.36^{a}$ \\
\hline$T$ at $1000 \mathrm{AU}(\mathrm{K})$ & 31 \\
\hline Density power-law index & 1.9 \\
\hline Density at $1000 \mathrm{AU}\left(\mathrm{g} \mathrm{cm}^{-3}\right)$ & $1.1 \times 10^{-19}$ \\
\hline$R_{\mathrm{env}}^{b}(\mathrm{AU})$ & $2.1 \times 10^{4}$ \\
\hline Envelope $\operatorname{mass}^{c}\left(M_{\odot}\right)$ & 6.0 \\
\hline
\end{tabular}

${ }^{a} 2 /(4+\beta) .{ }^{b}$ Radius for which the envelope density falls to a particle ambient density $n\left(\mathrm{H}_{2}\right)$ of $7.3 \times 10^{3} \mathrm{~cm}^{-3}$. ${ }^{c}$ Integrated up to $R_{\text {env }}$.

of the projected distance from the $1.2 \mathrm{~mm}$ peak (see Table 2). The result is plotted in Fig. 11 (top panel), together with the IRAM $30 \mathrm{~m}$ beam profile, and the best model fitted to the data.

In Table 10 we show the parameters of the envelope model, which have been calculated by following Estalella et al. (2009). In this paper the authors fit the radial intensity profile, using the full Planck function to describe the intensity, and the observed Spectral Energy Distribution (SED) simultaneously, adopting as a model of the source a spherically symmetric envelope of gas and dust surrounding the protostar(s). We do not include the possible contribution from the circumstellar disk because our angular resolution, $\sim 10^{\prime \prime}$ (8500 AU), is much larger than the typical sizes of accretion disks (tens to hundreds of AU). Note that we do not attempt to fit the near/mid-infrared emission of MM1, since it originates from components at higher temperatures than that responsible for the $\mathrm{mm} / \mathrm{submm}$ emission. So, the model is used to fit the SED up to frequencies corresponding to $60 \mu \mathrm{m}$. We assumed the dust opacity law 

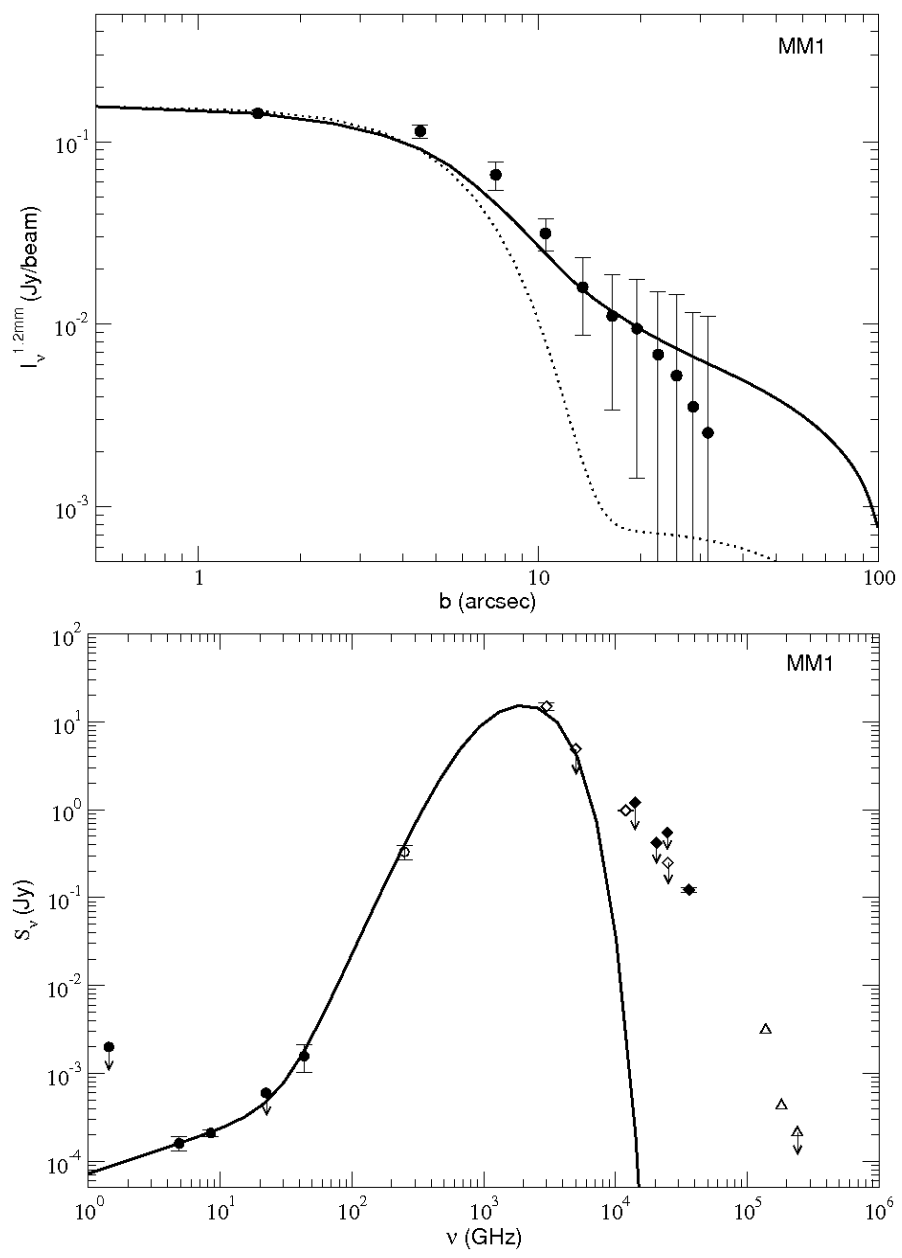

Fig. 11. Top: circularly averaged radial intensity profile at $1.2 \mathrm{~mm}$ of MM1. The error bars indicate the data rms inside each $3^{\prime \prime}$ wide ring. The dotted line shows the beam profile, including the error beams as given by Greve et al. (1998). The solid line shows the best fit model. Bottom: spectral energy distribution (SED) for MM1 in the IRAS 00213+6530 region. Filled circles are data from the VLA, the open circle represents the $1.2 \mathrm{~mm}(250 \mathrm{GHz})$ flux from the IRAM $30 \mathrm{~m}$ telescope, diamonds are IRAS data, filled diamonds are MSX data, and triangles are 2MASS data (see Table 11). The solid line is the sum of the free-free emission and envelope dust emission.

$\kappa_{v}=0.01(v / 230 \mathrm{GHz})^{\beta} \mathrm{cm}^{2} \mathrm{~g}^{-1}$ (Ossenkopf \& Henning 1994), $\beta$ being a free parameter of the model. For the density and temperature we considered power-laws as a function of radius, $\rho \propto r^{-p}$ and $T \propto r^{-q}$, with $p$ as a free parameter of the model, and $q=2 /(4+\beta)$ (Kenyon et al. 1993). In order to compare the model with the observed intensity profile, we computed the 2dimensional intensity map from the model, and we convolved it with the IRAM $30 \mathrm{~m}$ beam. We note that the beam was adopted to be the sum of two circular Gaussian beams. Then, from the convolved map, we computed the circularly averaged profile. Thus, our free parameters were the dust emissivity index $\beta$, the density power-law index $p$, and the scale of the density and temperature power-laws, namely, the density and temperature at a radius of $1000 \mathrm{AU}$ (taken arbitrarily as the reference radius for the power-laws). From the fitted parameters we derived the temperature power-law index $q$, the size, $R_{\mathrm{env}}$, and mass, $M_{\mathrm{env}}$, of the envelope. $R_{\text {env }}$ is defined as the radius for which the envelope density falls to a particle density similar to the ambient density, taken as $7.3 \times 10^{3} \mathrm{~cm}^{-3} . M_{\mathrm{env}}$ is the integral of the envelope mass density up to the envelope radius $R_{\text {env }}$ (see Table 10).
Table 11. Photometry of the MM1 clump.

\begin{tabular}{rrrl}
\hline \hline$\lambda$ & $\begin{array}{r}S_{v} \\
(\mathrm{mJy})\end{array}$ & $\begin{array}{r}F W H M \\
\left({ }^{\prime \prime}\right)\end{array}$ & $\begin{array}{l}\text { Survey } \\
\text { Instrument }\end{array}$ \\
\hline 1.25 & $<0.21^{a}$ & 2 & 2MASS \\
1.65 & $0.431 \pm 0.001^{a}$ & 2 & 2MASS \\
2.17 & $3.104 \pm 0.001^{a}$ & 2 & 2MASS \\
8.28 & $123 \pm 10^{b}$ & 18.3 & MSX \\
12 & $<250$ & 50 & IRAS \\
12.13 & $<549$ & 18.3 & MSX \\
14.65 & $<420$ & 18.3 & MSX \\
21.34 & $<1200$ & 18.3 & MSX \\
25 & $980 \pm 90$ & 50 & IRAS \\
60 & $<4910$ & 100 & IRAS \\
100 & $15000 \pm 1500$ & 230 & IRAS \\
1200 & $640 \pm 70$ & 11 & IRAM $30 \mathrm{~m}$ \\
7000 & $1.6 \pm 0.5^{c}$ & 3 & VLA \\
13000 & $<0.6^{c, d}$ & 1.5 & VLA \\
36000 & $0.27 \pm 0.02^{e}$ & 12 & VLA \\
60000 & $0.13 \pm 0.02^{e}$ & 15 & VLA \\
210000 & $<2.0^{d}$ & 45 & VLA \\
\hline
\end{tabular}

${ }^{a}$ J00241110+6547095. ${ }^{b}$ G120.1368+03.0617. ${ }^{c}$ VLA 8A. ${ }^{d} 4 \sigma$ upper limit. ${ }^{e}$ VLA 8 (VLA 8A and VLA 8B). ${ }^{f}$ From the NRAO VLA Sky Survey (Condon et al. 1998).

As mentioned above, the model fits the radial intensity profile and the SED simultaneously. The SED for MM1 was built by using the data shown in this work and by searching the literature for 2MASS, IRAC-Spitzer, MSX, and IRAS data. In Table 11 we list the photometry used for MM1 (adopting the values of VLA 8A for $1.3 \mathrm{~cm}, 7 \mathrm{~mm}$ and 2MASS, where the high angular resolution allows us to disentangle the different sources). We were able to simultaneously fit the SED and the intensity profile at $1.2 \mathrm{~mm}$. In Fig. 11 (bottom panel) we show the best fit of the model to the observed SED. In this figure we show the sum of the free-free emission and the envelope flux density integrated inside the radius of the envelope. The centimeter continuum emission is dominated by free-free emission with a spectral index of $0.5 \pm 0.4$. At millimeter and submillimeter wavelengths, the dust emission of the envelope is dominant.

The model resulting from the simultaneous fit to the radial intensity profile and the SED is able to fit the radial intensity profile remarkably well, but slightly underestimates the intensity at projected distances of $b=44^{\prime \prime} 5$ and 7".5 (see Fig 11 top panel). This can be due to the presence of several YSOs close $\left(<10^{\prime \prime}\right)$ to the $1.2 \mathrm{~mm}$ peak. The value obtained for the powerlaw density distribution index $p=1.9$ is similar to that found for other protostellar envelopes. Chandler \& Richer (2000) carried out a submillimeter survey of Class 0 and Class I sources and fitted the observed radial intensity profiles with density index $p$ between 1.5 and 2 for the majority of the sources. Hogerheijde $\&$ Sandell (2000) find $p=0.9-2.1$ in a sample of four Class I YSOs, and Motte \& André (2001) find $p=1.2-2.6$ in a sample of embedded YSOs in Taurus and Perseus, similar to the value obtained here.

\subsection{Column density maps and mass}

In order to estimate the relative $\mathrm{NH}_{3}$ abundance in I00213, we computed the $\mathrm{H}_{2}$ column density map. To do so, we used the expression given in Motte et al. (1998) to compute the column density of $\mathrm{H}_{2}$ from the $1.2 \mathrm{~mm}$ dust in a grid of 3 ". $3 \times 3$ '." 3 (the pixel size of our maps), and using a dust mass opacity coefficient 

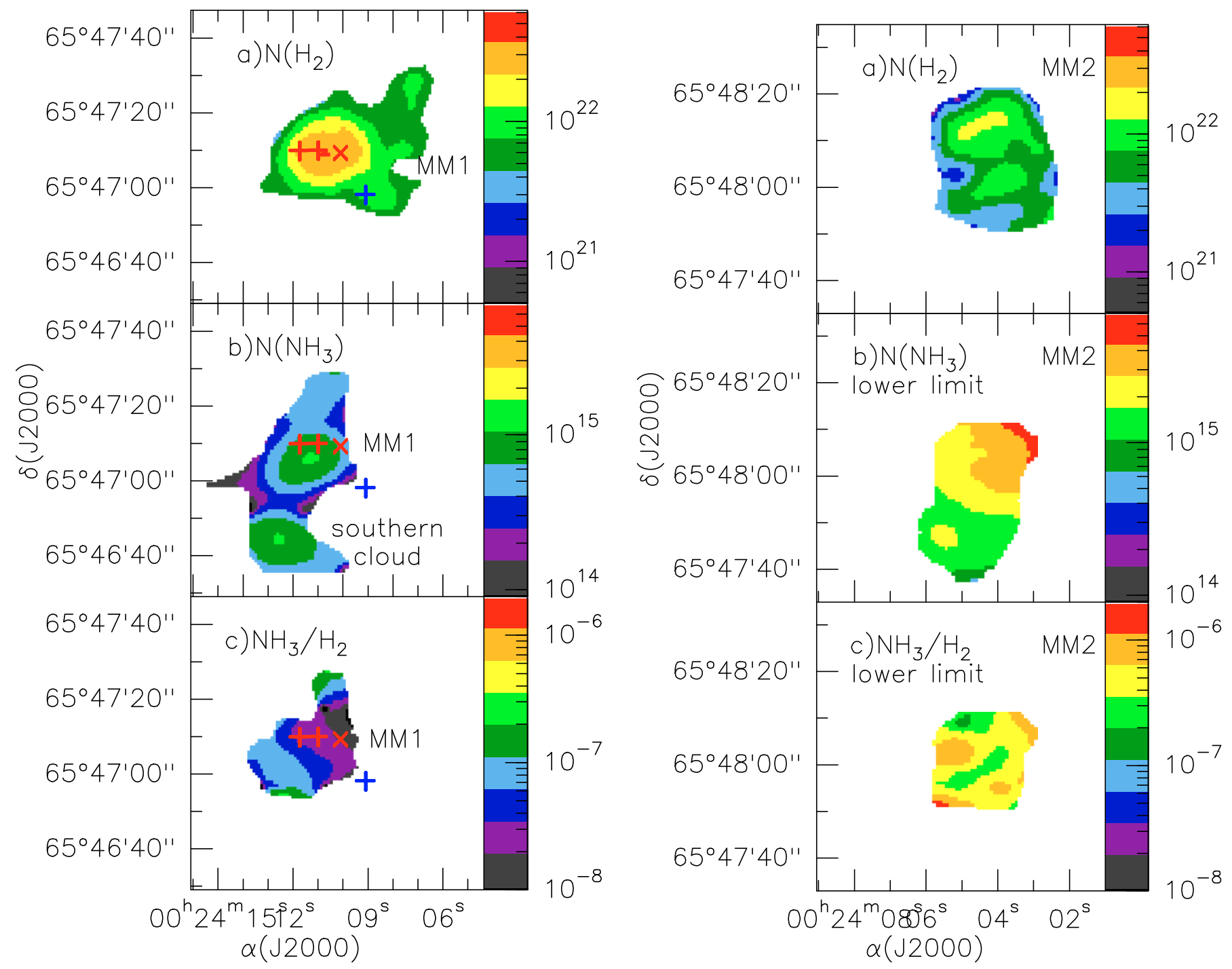

Fig. 12. a) $\mathrm{H}_{2}$ column density map from the $1.2 \mathrm{~mm}$ dust emission. b) $\mathrm{NH}_{3}$ column density map. c) $\mathrm{NH}_{3} / \mathrm{H}_{2}$ abundance map, for MM1 and the southern cloud (left panels) and for MM2 (right panels) in logarithmic scale. Scale units for the $\mathrm{H}_{2}$ and $\mathrm{NH}_{3}$ column density are $\mathrm{cm}^{-2}$. In all panels red crosses indicate the position of the two millimeter sources, VLA 8A and VLA 8B, and the red tilted cross marks the position of the infrared source IRS 1. The blue cross denotes the position of VLA 7. The color scale is the same for the left and right panels.

$\kappa_{1.2 \mathrm{~mm}}=0.01 \mathrm{~cm}^{2} \mathrm{~g}^{-1}$. Since the beam size of the $1.2 \mathrm{~mm}$ emission is a factor of 2 larger than the beam size of the $\mathrm{NH}_{3}$ observations, we convolved the $\mathrm{NH}_{3}$ emission to the same angular resolution as the $1.2 \mathrm{~mm}$ dust emission $\left(\sim 10^{\prime \prime}\right)$. We obtained the rotational temperature and column density of $\mathrm{NH}_{3}$ following the procedure described in Sect. 4.1 (see Appendix). In MM1 we obtained the rotational temperature from the $\mathrm{NH}_{3}(1,1)$ and $(2,2)$ emission, which was converted to kinetic temperature using the relation given in Tafalla et al. (2004, see also the Appendix). In order to estimate lower limits of the $\mathrm{NH}_{3}$ column density in the southern cloud and MM2 we adopted a rotational temperature of $10 \mathrm{~K}$ (see Sect. 5.2).

Maps of the $\mathrm{H}_{2}$ column density and $\mathrm{NH}_{3}$ column density are shown in Fig. 12. The $\mathrm{H}_{2}$ column density has a maximum value of $\sim 4 \times 10^{22} \mathrm{~cm}^{-2}$ toward the peak position of the $1.2 \mathrm{~mm}$ emission of MM1, and decreases in the more extended structure to values of $\sim 10^{22} \mathrm{~cm}^{-2}$. The uncertainty in the $\mathrm{H}_{2}$ column density is around a factor of 2, mainly due to the uncertainty in the dust opacity, as the uncertainty in the mm flux density (the rms of the map), and in the dust temperature constitute only a small contribution to the total uncertainty. The $\mathrm{NH}_{3}$ column density map, corrected for the primary beam response, also shows differences between the three $\mathrm{NH}_{3}$ clouds (see Fig. 12b). In MM1, the maximum value of the $\mathrm{NH}_{3}$ column density, $\sim 1.5 \times 10^{15} \mathrm{~cm}^{-2}$, is found to the south of VLA 8A and VLA 8B, which is consistent with the results found in Sect. 4.1. The uncertainty in the $\mathrm{NH}_{3}$ column density is of the order of $10-20 \%$. It has been estimated from the uncertainty in rotational temperature, in line width, and in $A \tau_{\mathrm{m}}$ (see Appendix). The values of the $\mathrm{NH}_{3}$ column density toward MM2 are significantly higher, in the range of $(2-7) \times 10^{15}$. For the case of MM2 and the southern cloud, the $\mathrm{NH}_{3}$ column density derived is a lower limit since the rotational temperature adopted is an upper limit due to the non-detection of $\mathrm{NH}_{3}(2,2)$.

We estimated the mass of each condensation from the $\mathrm{H}_{2}$ column density maps (see Table 12). The mass in MM1 is $\sim 3.5 M_{\odot}$, consistent with the values obtained from the $7 \mathrm{~mm}$ continuum emission and for the envelope model $\left(\sim 6 M_{\odot}\right)$. In addition, we compared the values obtained with the virial mass, $M_{\text {vir }}$, estimated using Eq. (5) of Beltrán et al. (2006), which assumes a spherical cloud with a power-law density distribution $\rho \propto r^{-p}$, with $p=2.0$, and neglecting contributions from magnetic fields and surface pressure. As can be seen in Table 12, the mass of MM1 is higher than the virial mass, indicating that it 
Table 12. Physical parameters of IRAS $00213+6530$.

\begin{tabular}{lrccc}
\hline \hline Region & $\begin{array}{c}\operatorname{Mass}^{a} \\
\left(M_{\odot}\right)\end{array}$ & $\begin{array}{c}\Delta v^{b} \\
\left(\mathrm{~km} \mathrm{~s}^{-1}\right)\end{array}$ & $\begin{array}{c}\theta^{c} \\
(\operatorname{arcsec})\end{array}$ & $\begin{array}{c}M_{\text {vir }}^{d} \\
\left(M_{\odot}\right)\end{array}$ \\
\hline MM1 & 3.5 & 0.85 & 7 & 1.3 \\
Southern cloud & $<0.3$ & 0.65 & 6 & 0.7 \\
MM2 & 1.1 & 0.75 & 11 & 1.6 \\
\hline
\end{tabular}

${ }^{a}$ Estimated as $M=\mu_{\mathrm{H}_{2}} m_{\mathrm{H}} \sum N\left(\mathrm{H}_{2}\right) \Delta A$, where $N\left(\mathrm{H}_{2}\right)$ is taken from the $\mathrm{H}_{2}$ column density map, $\Delta A$ is the pixel area, and a mean molecular mass per $\mathrm{H}_{2}$ molecule $\mu_{\mathrm{H}_{2}}=2.8$, which corresponds to a $10 \%$ helium abundance. ${ }^{b}$ Intrinsic line width of the magnetic hyperfine components.

${ }^{c}$ Deconvolved geometrical mean size of the major and minor axes of the source, obtained from a 2D Gaussian fit. ${ }^{d}$ Estimated for a density distribution $n \propto r^{-2}$.

might be unstable and undergoing collapse. The mass of MM2 is $M \simeq 0.7 M_{\mathrm{vir}}$, indicating that the material in this cloud is stable. In contrast, toward the southern cloud the total mass of gas is $M<0.4 M_{\mathrm{vir}}$, this clump being gravitationally unbound and it could disperse at roughly the internal sound speed of $\sim 0.3 \mathrm{~km} \mathrm{~s}^{-1}$ on a timescale of around $10^{5} \mathrm{yr}$, unless it is confined by external pressure. All this suggests that the southern cloud could be in an earlier evolutionary stage than MM1 and MM2.

\subsection{Relative $\mathrm{NH}_{3}$ abundance}

In Fig. 12c we show the relative $\mathrm{NH}_{3}$ abundance maps of MM1 and MM2. The typical value of the $\mathrm{NH}_{3}$ abundance found in MM1, in a position just south of the three YSOs, is around $2 \times 10^{-8}-4 \times 10^{-8}$, which is similar to the typical value found in dense clouds (Herbst \& Klemperer 1973; see Anglada et al. 1995, for a discussion on $\mathrm{NH}_{3}$ abundances). In MM1, near the YSOs, there is a slight trend in the $\mathrm{NH}_{3}$ abundance to increase, from $1 \times 10^{-8}$ near IRS 1 , up to $3 \times 10^{-8}$ toward VLA $8 \mathrm{~B}$. In this case, the most evolved YSO IRS 1 is likely dispersing the dense gas material of its surroundings, and the result is a decrease of the $\mathrm{NH}_{3}$ abundance. It is, however, a modest effect, slightly above the typical uncertainty of a factor of 2 . Toward the southern cloud we derived a lower limit for the relative $\mathrm{NH}_{3}$ abundance of $\sim(1-4) \times 10^{-7}$.

Regarding the $\mathrm{NH}_{3}$ abundance in MM2, we find values ranging from $1-2 \times 10^{-7}$ up to $1 \times 10^{-6}$. This abundance is higher than in MM1, despite the observed $\mathrm{NH}_{3}$ emission in MM2 being fainter than in MM1, mainly due to two effects: the $\mathrm{NH}_{3}$ column density is higher due to the correction for the primary beam response, and the $\mathrm{H}_{2}$ column density is low, $\sim 3 \times 10^{21}-1 \times 10^{22} \mathrm{~cm}^{-2}$. The relative $\mathrm{NH}_{3}$ abundance in MM2 is a lower limit. Although these abundances are high, Benson $\&$ Myers (1983) and Ohishi et al. (1992) find $\mathrm{NH}_{3}$ abundances around $\sim 2 \times 10^{-7}$ in starless cores of the dark clouds L1498 and L1512, and in L134N, respectively. In addition, chemical models of Hartquist et al. (2001) are able to reproduce such a high $\mathrm{NH}_{3}$ abundance for a young core, Core D in TMC-1, with $\mathrm{N}_{2}$ and $\mathrm{CO}$ not freezing-out. Therefore, it is plausible that MM2 and the southern cloud are in a young evolutionary stage, as suggested by the starless properties of this cloud (see Sect. 5.2).

\section{Discussion}

The results obtained for IRAS $00213+6530$ show that in this region there are different sources, which have different radio and infrared properties.

\subsection{VLA 8A, VLA 8B, and IRS 1: multiple sources in different evolutionary stages}

The centimeter and millimeter continuum observations, together with near-infrared data, allowed us to identify three YSOs in MM1: IRS 1, VLA 8A and VLA 8B. While at $3.6 \mathrm{~cm}$ we could not resolve the centimeter emission of VLA 8 , at $7 \mathrm{~mm}$ we found two sources, VLA 8A and VLA 8B. In the near infrared, only VLA 8A and IRS 1 are detected. In addition, all sources are deeply embedded in the dense gas traced by $\mathrm{NH}_{3}$. Line broadening and local heating have been detected toward their position, indicating the true association of the three objects with the molecular gas.

From these preliminary results, we can make a rough estimate of the evolutionary stage of the detected sources. First, IRS 1 is associated with an infrared source with no detected $7 \mathrm{~mm}$ continuum emission. VLA 8A, although bright in the infrared, is the strongest millimeter source in the field. Its SED has a quite steep profile at the 2MASS wavelengths. Thus, $\mathrm{d}\left(\log \lambda F_{\lambda}\right) / \mathrm{d}(\log \lambda)>0$ between 1 and $10 \mu \mathrm{m}$, which is consistent with the classification of VLA $8 \mathrm{~A}$ as a Class I source (e.g., Hartmann 1998). Regarding the near-infrared colors of the 2MASS sources associated with IRS 1 and VLA 8A, derived from 2MASS photometry (see Table 13), we found that both sources fall inside the area corresponding to YSOs of Class 0/I (e.g., Itoh et al. 1996; Ojha et al. 2004; Matsuyanagi et al. 2006). Finally, VLA 8B, which is also associated with dense gas tracers, shows no infrared emission at all. At $7 \mathrm{~mm}$, VLA 8B has associated little dust continuum emission, suggesting that the dust emission is colder, probably detectable at $1 \mathrm{~mm}$ and/or submillimeter wavelengths. Thus, VLA 8B could be in a previous stage of evolution, being still more embedded than VLA 8A, possibly in the Class 0 phase. We found an extended temperature enhancement to the north of VLA 8A and VLA 8B, which seems to be associated with the passage of an outflow that heats and perturbs the dense gas; Busquet et al. (in prep.) find that the large scale molecular outflow (Yang et al. 1990), when observed with high angular resolution, is centered on a position near VLA 8A and VLA 8B, these sources being candidates to drive the observed high-velocity gas.

Thus, it seems that the I00213 region harbors a multiple system of low mass protostars, indicating that the star formation process in this region does not produce only a single YSO. Since low mass protostars evolve approximately at the same rate to the main sequence, the different evolutionary stages found in I00213 suggest that stars in this region are not forming simultaneously but continuously. Also, there may be different generations due to different timescales of core collapse, as has been found in other low mass star-forming regions (e.g., L1551: Moriarty-Schieven et al. 2006), indicating that the formation of different stars is not simultaneous but sequential in time, possibly triggered by the interaction of the molecular outflow with a dense core in its surroundings (Yokogawa et al. 2003; Shimajiri et al. 2008). Therefore, the initial assumption that star formation occurs in an isolated mode may not be appropriate to describe the I00213 region when the region is studied with high angular resolution. This poses the question of to what extent we can adopt the isolated mode in the theories of star formation, as this high angular resolution study together with a large number of recent studies (e.g., Huard et al. 1999; Djupvik et al. 2006; Teixeira et al. 2007; Carrasco-González et al. 2008; Chen et al. 2008, 2009; Forbrich et al. 2009; Gutermuth et al. 2008; Swift \& Welch 2008; Girart et al. 2009) suggest that isolated star formation seems to be rare in the Galaxy, even in low mass star-forming regions. 
Table 13. Infrared excess of 2MASS sources.

\begin{tabular}{lccc}
\hline \hline 2MASS & Associated & & \\
Source & Source & $(J-H)$ & $(H-K)$ \\
\hline J00241110+6547095 & VLA 8A & $1.02 \pm 0.16$ & $1.47 \pm 0.18$ \\
J00241010+6547091 & IRS 1 & $1.25 \pm 0.26$ & $2.61 \pm 0.27$ \\
J00241251+6546418 & $\ldots$ & $0.48 \pm 0.04$ & $0.12 \pm 0.05$ \\
\hline
\end{tabular}

The three YSOs are spatially ordered from youngest (east) to oldest (west), suggesting that an external agent could be inducing star formation in MM1. For this, it would be very useful to identify and map the molecular outflows in the region. Alternatively, it would be very useful as well to study the possible association of I00213 with the HII region S171, located to the northwest of I00213. Finally, it is worth noting that the I00213 region falls exactly on the southern border of the Cep OB4 shell (Kun 2008).

\subsection{Starless candidates: MM2 and the southern cloud}

While the southern cloud is detected only in $\mathrm{NH}_{3}, \mathrm{MM} 2$ is detected both in $\mathrm{NH}_{3}$ and dust emission. Given that the $(2,2)$ line is not detected toward the southern cloud, and only detected toward the northern peak of MM2, these clouds are cold, since $T_{\text {rot }}<11 \mathrm{~K}$. As mentioned in Sect. 3.3, the nearinfrared source 2MASS J00241251+6546418 spatially coinciding with the southern cloud is not likely associated with the dense gas. We estimated the infrared excess from the $(J-H)$ vs. $(H-J)$ diagram (see Table 13). The near-infrared colors derived are characteristic of main sequence stars, giants, supergiants, Class III sources, or Class II sources with small infrared excess (Itoh et al. 1996; Matsuyanagi et al. 2006). In addition, this source has an optical counterpart seen in the DSS2 image. Thus, it is likely a foreground source, not associated with the $\mathrm{NH}_{3}$ dense gas and the I00213 star-forming region. No near-infrared sources are associated with MM2. In addition, neither MM2 nor the southern cloud seem to be associated with molecular outflows (Busquet et al. in prep.). Therefore, no clear signposts of stellar activity are found for these two clouds, suggesting that they could be starless.

\subsection{On the nature of VLA 7}

VLA 7, lying outside the $\mathrm{NH}_{3}$ dense gas and dust emission, has a very negative spectral index at centimeter wavelengths $(-1.6 \pm 0.2)$, indicating that the emission has a non-thermal origin, found typically for extragalactic sources with a steep spectrum and some pulsars (e.g., Lehtinen et al. 2003). Given the close proximity to MM1, we considered the possibility that VLA 7 could be related to the I00213 region. In low mass starforming regions, non-thermal emission has been detected toward some YSOs, like Class 0 YSOs (Choi et al. 2008) or T-Tauri stars (e.g., André 1996; Rodríguez et al. 1999; Gibb 1999). However, centimeter emission arising from weak T-Tauri stars is usually polarized, and they are often optically visible. We did not find evidence of circular polarized emission toward VLA 7, neither a visible counterpart, so we consider unlikely this possibility. Another possibility to explain the negative spectral index of VLA 7 is non-thermal synchrotron emission produced in shocked regions of outflowing gas, found mainly in high mass star-forming regions (e.g., Rodríguez \& Reipurth 1989; Garay et al. 2003; Rodríguez et al. 2005). As there is molecular outflow emission in this region (Busquet et al. in prep.) we cannot rule out this possibility, and further observations would help to confirm the true association of VLA 7 with the I00213 star-forming region.

\section{Conclusions}

We observed with the VLA, IRAM 30 m Telescope, and the NASA $70 \mathrm{~m}$ antenna at Robledo de Chavela (Spain) the continuum emission at $6 \mathrm{~cm}, 3.6 \mathrm{~cm}, 1.3 \mathrm{~cm}, 7 \mathrm{~mm}$, and $1.2 \mathrm{~mm}$, the $\mathrm{NH}_{3}(1,1)$ and $\mathrm{NH}_{3}(2,2)$ lines, and the $\mathrm{H}_{2} \mathrm{O}$ maser and CCS emission toward the low mass star-forming region IRAS $00213+6530$. Our main conclusions can be summarized as follows:

1. The $1.2 \mathrm{~mm}$ continuum emission observed with the IRAM 30 m shows two dust condensations, MM1 and MM2. The continuum emission at centimeter and millimeter wavelengths, together with the available data from 2MASS, have revealed three sources, IRS 1, VLA 8A, and VLA 8B, all embedded in the dusty cloud MM1. These sources show different radio and infrared properties, and seem to be in different evolutionary stages, with VLA 8B being in the earliest phase. In MM1, low mass star formation appears to proceed along a west-east direction.

2. We marginally detected $\mathrm{H}_{2} \mathrm{O}$ maser emission toward $\mathrm{I} 00213$ with the NASA $70 \mathrm{~m}$ antenna during the observations carried out on 2008 September 23, but other attempts, including the VLA observations, yielded negative results.

3. The YSOs found in the region are deeply embedded in the high-density gas. The $\mathrm{NH}_{3}(1,1)$ emission traces an elongated structure that consists of a main cloud (MM1 and the southern cloud) and a smaller cloud, MM2, located to the northwest of MM1. While the southern cloud and MM2 appear as quiescent and starless, in MM1 there is evidence of a perturbation of the gas (line broadening and local heating) along the east-west direction, associated with IRS 1 and north of the two millimeter sources, elongated in the northsouth direction. We propose that part of the dense gas is being perturbed by the passage of one or more outflow(s).

4. We detected CCS emission toward I00213 using the NASA $70 \mathrm{~m}$ antenna with a line width, $\sim 1.3 \mathrm{~km} \mathrm{~s}^{-1}$, that is large compared with previous studies.

5. The source VLA 7, which has a negative spectral index, lies outside but near the border of the $\mathrm{NH}_{3}(1,1)$ condensation. Although this source could be a background source we cannot rule out the possibility that VLA 7 could be the result of the interaction of a molecular outflow with the surrounding medium.

6. We used a spherically symmetric envelope model that simultaneously fits the observed SED from $7 \mathrm{~mm}$ to $60 \mu \mathrm{m}$, and the radial intensity profile at $1.2 \mathrm{~mm}$ of the clump associated with MM1. The best fit was obtained for a dust opacity law index $\beta=1.5$, a temperature at $1000 \mathrm{AU}$ of $31 \mathrm{~K}$, and a density at $1000 \mathrm{AU}$ of $1.1 \times 10^{-19} \mathrm{~g} \mathrm{~cm}^{-3}$ or a particle density of $n\left(\mathrm{H}_{2}\right) \sim 2.3 \times 10^{4} \mathrm{~cm}^{-3}$. The envelope radius is $R_{\text {env }} \sim 21000 \mathrm{AU}\left(3.1 \times 10^{17} \mathrm{~cm}\right)$, and inside this radius the envelope model mass is $6 M_{\odot}$.

7. There is a strong differentiation of $\mathrm{NH}_{3}$ abundance in the region. In particular, we found low values, $\sim 2 \times 10^{-8}$, of the $\mathrm{NH}_{3}$ abundance associated with MM1, which contains the YSOs. On the other hand, toward those clouds with starless properties (the southern cloud and MM2), the $\mathrm{NH}_{3}$ abundance rises to $\sim 1 \times 10^{-6}$, suggesting that in evolved clouds 
with star-formation activity there is a decrease in the $\mathrm{NH}_{3}$ abundance.

Acknowledgements. G.B. is grateful to Serena Viti, David A. Williams, and Oscar Morata for useful discussion on the ammonia abundance. We are grateful to the anonymous referee and to the editor for valuable comments. The authors are supported by the Spanish MEC grant AYA2005-08523-C03, and the MICINN grant AYA2008-06189-C03 (co-funded with FEDER funds). A.P. is also supported by the MICINN grant ESP2007-65475-C02-02 and the program ASTRID S0505/ESP-0361 from La Comunidad de Madrid and the European Social Fund. G.A. acknowledges support from Junta de Andalucía. This publication makes use of the data products from the Two Micron All Sky Survey, which is a joint project of the University of Massachusetts and the Infrared Processing and Analysis Center/California Institute of Technology, funded by the National Aeronautics and Space Administration (NASA) and the National Science Foundation.

\section{References}

Adams, F. C., \& Myers, P. C. 2001, ApJ, 553, 744

André, P. 1996, in Radio Emission from the Stars and the Sun, ed. A. R. Taylor, \& J. M. Paredes (San Francisco: ASP), ASP Conf. Ser., 93, 273

Anglada, G., Estalella, R., Mauersberger, J., et al. 1995, ApJ, 443, 682

Anglada, G., Sepúlveda, I., \& Gómez, J. F. 1997, A\&AS, 121, 255

Anglada, G., Villuendas, E., Estalella, R., et al. 1998, ApJ, 116, 2953

Beltrán, M. T., Estalella, R., Anglada, G., Rodríguez, L. F., \& Torrelles, J. M. 2001, ApJ, 121, 1556

Beltrán, M. T., Girart, J. M., \& Estalella, R. 2006, A\&A, 457, 865

Benson, P. J., \& Myers, P. C. 1983, ApJ, 270, 589

Benson, P. J., \& Myers, P. C. 1989, ApJS, 71, 89

Briggs, D. 1995, Ph.D. Thesis, New Mexico Inst. of Mining and Technology

Brooke, T. Y., Huard, T. L., Bourke, T. L., et al. 2007, ApJ, 655, 364

Carilli, C. L., \& Holdaway, M. A. 1997, Millimeter Array Technical Memo, 173 (NRAO)

Carrasco-González, C., Anglada, G., Rodríguez, L. F., et al. 2008, ApJ, 676 1073

Chandler, C. J., \& Richer, J. S. 2000, ApJ, 530, 851

Chen, X., Bourke, T. L., Launhardt, R., \& Henning, T. 2008, ApJ, 686, L107

Chen, X., Launhardt, R., \& Henning, T. 2009, ApJ, 691, 1729

Choi, M., Hamaguchi, K., Lee, J-.E., \& Tatematsu, K. 2008, ApJ, 687, 406

Clarke, C. J., Bonnell, I. A., \& Hillenbrand. L. A. 2000, in Protostars \&

Planets IV, ed. V. Mannings, A. P. Boss, \& S. S. Russell (Tucson: University of Arizona Press), 151

Condon, J. J., Cotton, W. D., Greisen, E. W., et al. 1998, AJ, 115, 1693

de Gregorio-Monsalvo, I., Chandler, C. J., Gómez, J. F., et al. 2005, ApJ, 628, 789

de Gregorio-Monsalvo, I., Gómez, J. F., Suárez, O., et al. 2006, ApJ, 642, 319

Djupvik, A. A., André, Ph., Bontemps, S., et al. 2006, A\&A, 458, 789

Estalella, R., Palau, A., Girart, J. M., et al. 2009, A\&A, submitted

Felli, M., Palagi, F., \& Tofani, G. 1992, A\&A, 255, 293

Forbrich, J., Stanke, T., Klein, R., et al. 2009, A\&A, 493, 547

Garay G., Brooks K. J., Mardones D., \& Norris R. P. 2003, ApJ, 587, 739

Gibb, A. G. 1999, MNRAS, 304, 1

Girart, J. M., Estalella, R., Ho, P. T. P., \& Rudolph, A. L. 2000, ApJ, 539, 763

Girart, J. M., Rao, R., \& Estalella, R. 2009, ApJ, 694, 56

Gómez, M., Hartmann, L., Kenyon, S. J., \& Hewett, R. 1993, AJ, 105, 1927

Greve, A., Kramer, C., \& Wild, W. 1998, A\&AS, 133, 271

Gutermuth, R. A., Bourke, T. L., Allen, L. E., et al. 2008, ApJ, 673, L151

Han, F., Mao, R. Q., Lu, J., et al. 1998, A\&AS, 127181

Harju, J., Walmsley, C. M., \& Wouterloot, J. G. A. 1993, A\&AS, 98, 51

Hartmann, L. 1998, in Accretion Processes in Star Formation, ed. A. King, D.

Lin, S. Maran, J. Pringle, \& M. Ward (Cambrigde: Cambridge University Press), 8

Hartquist, T. W., Williams, D. A., \& Viti, S. 2001, A\&A, 369, 605

Herbst, E., \& Klemperer, W. 1973, ApJ, 185, 505

Ho, P. T. P., \& Townes, C. H. 1983, ARA\&A, 21, 239

Hogerheijde, M. R., \& Sandell, G. 2000, ApJ, 534, 880

Huard, T. L., Sandell, G., \& Weintraub, D. A. 1999, ApJ, 526, 833

Itoh, Y., Tamura, M., \& Gatley, I. 1996, ApJ, 465, L129

Kenyon, S. J., Calvet, N., \& Hartmann, L. 1993, ApJ, 414, 676

Kun, M. 2008, Star Forming Regions in Cassiopeia, Handbook of Star Forming Regions, V. I: The Northern Sky, ASP Monograph Publications, ed. by B. Reipurth, 4, 240

Lada, C. J. 1999, in The Origin of Stars and Planetary Systems, ed. C. J. Lada, \& N. D. Kylafis (Kluwer Acad. Publ.), 143

Lada, C. J., \& Lada, E. A. 2003, ARA\&A, 41, 57
Lada, E. A., Strom, K. M., \& Myers, P. C. 1993, in Protostars \& Planets III, ed. E. H. Levy, \& J. I. Lumine (Tucson: University of Arizona Press), 245 Lee, J.-E., Di Francesco, J., Lai, S.-P, et al. 2006, ApJ, 648, 49

Lehtinen, K., Harju, J., Kontinen, S., \& Higdon, J. L. 2003, A\&A, 401, 1017

Matsuyanagi, I., Itoh, Y., Sugitani. K., et al. 2006, PASJ, 58, L29

Moriarty-Schieven, G. H., Johnstone, D., Bally, J., \& Jenness, T. 2006, ApJ, 645, 357

Motte, F., \& André, P. 2001, A\&A, 365, 440

Motte, F., André, P., \& Neri, R. 1998, A\&A, 336, 150

Ohishi, M., Irvine, W. M., \& Kaifu, N. 1992, in Astrochemistry of Cosmic Phenomena, ed. P. D. Singh (Dordrecht: Kluwer), 171

Ojha, D. K., Tamura, M., Nakajima, Y., \& Fukagawa M. 2004, ApJ, 608, 797 Ossenkopf, V., \& Henning, Th. 1994, A\&A, 291, 943

Pauls, A., Wilson, T. L., Bieging, J. H., \& Martin, R. N. 1983, A\&A, 124, 23

Pfalzner, S., Tackenberg, J., \& Steinhausen, M. 2008, A\&A, 487, L45

Rodríguez, L. F., Anglada, G., \& Curiel, S. 1999, ApJS, 125, 427

Rodríguez L. F., Garay G., Brooks K. J., \& Mardones D. 2005, ApJ, 626, 953

Rodríguez L. F., \& Reipurth, B. 1989, Rev. Mex. Astron. Astrofis., 17, 59

Sepúlveda, I. 1993, Master Thesis. University of Barcelona

Sepúlveda, I. 2001, Ph.D. Thesis. Universitat de Barcelona

Shimajiri, Y., Takahashi, S., Takakuwa, S., Saito, M., \& Kawabe, R. 2008, ApJ, 683,255

Shu, F. H., Adams, F. C., \& Lizano, S. 1987, ARA\&A, 25, 23

Sweitzer, J. S. 1978, ApJ, 225, 116

Swift, J. J., \& Welch, W. J. 2008, ApJS, 174, 202

Tafalla, M., Myers, P. C., Caselli, P., \& Walmsley, C. M. 2004, A\&A, 416, 191

Teixeira, P. S., Zapata, L. A., \& Lada, C. J. 2007, ApJ, 667, L179

Ungerechts, H., Winnewisser, G., \& Walmsley, C. M. 1986, A\&A, 157, 207

Yang, J., Fukui, Y., Umemoto, T., \& Ogawa, H. 1990, ApJ, 362, 538

Yokogawa, S., Kitamura, Y., Momose, M., \& Kawabe, R. 2003, ApJ, 595, 266

\section{Appendix A: Derivation of $T_{\text {rot }}$ and $N\left(\mathrm{NH}_{3}\right)$ from $\mathrm{NH}_{3}(1,1)$ and $(2,2)$ observations}

\section{$T_{\mathrm{MB}}(1,1 ; m)$ and $N(1,1)$ :}

The $\mathrm{NH}_{3}(1,1)$ method of CLASS fits the magnetic hyperfine structure of $\mathrm{NH}_{3}(1,1)$. The output parameters for the fit to the hyperfine structure are: $A \tau_{\mathrm{m}}$, the velocity of the reference line, the intrinsic line width, and the optical depth of the (1, 1) main line, $\tau_{m}$ (sum of the optical depths of the magnetic hyperfine components of the main line), $\tau_{\mathrm{m}}=\tau(1,1) / 2$. The parameter $A$, according to Pauls et al. (1983), is defined as $A=f\left[J_{v}\left(T_{\text {ex }}\right)-J_{v}\left(T_{\mathrm{bg}}\right)\right]$, where $f$ is the filling factor. Then, from the output parameters, and applying the radiative transfer equation, one can obtain the $(1,1)$ main line temperature $T_{\mathrm{MB}}(1,1 ; m)$,

$T_{\mathrm{MB}}(1,1 ; m)=A \tau_{\mathrm{m}} \frac{1-\mathrm{e}^{-\tau_{\mathrm{m}}}}{\tau_{\mathrm{m}}}$.

The excitation temperature $T_{\mathrm{ex}}$ is not directly obtained from the fit, but is isolated from the output parameter $A \tau_{\mathrm{m}}$,

$$
T_{\mathrm{ex}}=\frac{1.14}{\ln \left(1+1.14 /\left[A \tau_{\mathrm{m}} / \tau_{\mathrm{m}}+J_{v}\left(T_{\mathrm{bg}}\right)\right]\right)} .
$$

Note that no assumption is made concerning $T_{\mathrm{ex}}$ with respect to the background temperature $T_{\mathrm{bg}}$.

The beam averaged column density in the $(1,1)$ level (Anglada et al. 1995),

$$
\left[\frac{N(1,1)}{\mathrm{cm}^{-2}}\right]=1.58 \times 10^{13} f \frac{\mathrm{e}^{1.14 / T_{\mathrm{ex}}}+1}{\mathrm{e}^{1.14 / T_{\mathrm{ex}}}-1} \tau_{\mathrm{m}}\left[\frac{\Delta v}{\mathrm{~km} \mathrm{~s}^{-1}}\right],
$$

the filling factor $f$ being assumed to be 1 for our VLA observations.

To derive Eq. (A.3), $N(1,1)$ is not approximated to $2 N_{+}(1,1)$, but is taken as $N(1,1)=N_{+}(1,1)\left[1+\exp \left(h v_{11} / k T_{\text {ex }}\right)\right]$ (see Harju et al. 1993, for more details). 
$T_{\mathrm{MB}}(2,2 ; m)$ :

For $\mathrm{NH}_{3}(2,2)$ we fitted one single Gaussian, with the $(2,2)$ main line temperature, $T_{\mathrm{MB}}(2,2 ; m)$, being an output parameter of the fit.

$T_{\text {rot }}^{21}$ :

The rotational temperature derived from $\mathrm{NH}_{3}(1,1)$ and $\mathrm{NH}_{3}(2$, 2) can be estimated, following Ho \& Townes (1983, Eq. (4)), by assuming that the transitions between the metastable inversion doublets are approximated as a two-level system, and that the excitation temperature $T_{\mathrm{ex}}$ and line width $\Delta v$ are the same for both $\mathrm{NH}_{3}(1,1)$ and $\mathrm{NH}_{3}(2,2)$. Then,

$T_{\text {rot }}^{21}=\frac{-41.5}{\ln \left(-\frac{0.283}{\tau_{\mathrm{m}}} \ln \left[1-\frac{T_{\mathrm{MB}}(2,2 ; m)}{T_{\mathrm{MB}}(1,1 ; m)} \cdot\left(1-\mathrm{e}^{-\tau_{\mathrm{m}}}\right)\right]\right)}$.

Note that we did not assume that the emission is optically thin. The assumption of a two-level system is reasonable because transitions between the metastable inversion doublets are usually much faster than those of other rotational states (Ho \& Townes 1983). If the density and temperature were high enough to populate the upper non metastable states, multilevel statistical calculations would be required (e.g., Sweitzer et al. 1978).

An estimate of the gas kinetic temperature can be obtained by correcting the rotational temperature derived from $\mathrm{NH}_{3}$, using the expression given in Tafalla et al. (2004),

$T_{\mathrm{k}}=\frac{T_{\mathrm{rot}}^{21}}{1-\frac{T_{\mathrm{rot}}^{21}}{42} \ln \left[1+1.1 \mathrm{e}^{-16 / T_{\mathrm{rot}}^{21}}\right]}$,

which is almost independent of core density and size. This relation is recommended for the range $T_{\mathrm{k}}=5-20 \mathrm{~K}$.

\section{$N\left(\mathrm{NH}_{3}\right)$ :}

The $\mathrm{NH}_{3}$ column density was derived by following Ungerechts et al. (1986), and Harju et al. (1993). The main assumptions are: i) only metastable levels are populated; ii) $T_{\text {rot }}$ is the same for each pair of rotational levels; iii) the ratio of the column densities of each rotational level is the same as the ratio of the column densitiesof upper inversion levels; $i v$ ) the contribution to the total $\mathrm{NH}_{3}$ column density comes essentially from levels with $J \leq 3$; $v$ )the relative population of all metastable levels of both ortho and para- $\mathrm{NH}_{3}$ is that given by thermal equilibrium at temperature $T_{\text {rot }}$; and $v i$ ) the frequencies for the $\mathrm{NH}_{3}(1,1)$ and $\mathrm{NH}_{3}(2,2)$ transitions are very similar. With these assumptions,

$N\left(\mathrm{NH}_{3}\right)=N(1,1)\left[\frac{1}{3} \mathrm{e}^{23 \cdot 4 / T_{\text {rot }}^{21}}+1+\frac{5}{3} \mathrm{e}^{-41.5 / T_{\text {rot }}^{21}}+\frac{14}{3} \mathrm{e}^{-101.2 / T_{\text {rot }}^{21}}\right]$.

\section{Uncertainty in $T_{\mathrm{rot}}^{21}$ :}

In order to estimate the uncertainties associated with $T_{\text {rot }}$ and $N\left(\mathrm{NH}_{3}\right)$ introduced by this method, we did the following.

The error of $T_{\text {rot }}^{21}$ was estimated by assuming optically thin emission and that the main sources of error come from $T_{\mathrm{MB}}(1,1 ; m)$ and $T_{\mathrm{MB}}(2,2 ; m)$. Defining $R \equiv T_{\mathrm{MB}}(2,2 ; m) / T_{\mathrm{MB}}(1,1 ; m)$, the relative error is $\frac{\delta R}{R}=\sqrt{\left(\frac{\delta T_{\mathrm{MB}}(1,1 ; m)}{T_{\mathrm{MB}}(1,1 ; m)}\right)^{2}+\left(\frac{\delta T_{\mathrm{MB}}(2,2 ; m)}{T_{\mathrm{MB}}(2,2 ; m)}\right)^{2}}$, with $\delta T_{\mathrm{MB}}(1,1 ; m)$ and $\delta T_{\mathrm{MB}}(2,2 ; m)$ given directly by the hyperfine fit. Then, the error in the rotational temperature was estimated as

$\delta T_{\mathrm{rot}}^{21}=\frac{-41.5}{\ln ^{2}(0.283 R)} \frac{\delta R}{R}$.

As a test for the previous estimate of the error in $T_{\text {rot }}$, we estimated the opacity from the ratio of the main line intensity to the inner satellite average intensity, $T_{\mathrm{MB}}(1,1, i s)$, following Ho \& Townes (1983),

$\frac{T_{\mathrm{MB}}(1,1 ; m)}{T_{\mathrm{MB}}(1,1 ; i s)}=\frac{1-\mathrm{e}^{-\tau_{\mathrm{m}}}}{1-\mathrm{e}^{-\tau_{\mathrm{m}} / 3.6}}$,

and derived $T_{\text {rot }}$ with this estimate of the opacity. The opacities derived from this method are systematically lower than but compatible to the values derived from the hyperfine fit, and the rotational temperature obtained agrees with the values derived from the $\mathrm{NH}_{3}(1,1)$ hyperfine method used in this work. We note that the opacity inferred from the hyperfine fit seems to be more reliable than the opacity from the ratio of the main line to the satellites when the width of the magnetic hyperfine components is comparable to the their separation in velocity (e.g., Anglada et al. 1995), which is $0.11-0.53 \mathrm{~km} \mathrm{~s}^{-1}$. This is probably the case of our region. However, since the observations reported here were carried out with a spectral resolution in some cases lower than the intrinsic line width, the hyperfine fits must be regarded with caution and for this reason we compared them with the ratio of the main line to the satellite method. 